\title{
LA POSIZIONE DEL CAPITOLO IX DEL PRINCIPE NEL PENSIERO DI MACHIAVELLI
}

\author{
di Giovanni G. Balestrieri
}

Non si corre di certo il rischio di sbagliare a dire che il principato civile, tema del IX capitolo del Principe, sia assurto al rango di attore protagonista del pensiero machiavelliano, richiamando su di sé nel corso degli ultimi decenni le cure e l'attenzione della critica. A nessuno sembra oggi consentito, giunto al suo cospetto, minimizzarne la portata, assorbendone il contenuto entro la cornice complessiva del trattato, misconoscendone, quindi, la posizione di preminenza nell'elaborazione di Machiavelli. Pure, riesce difficile immaginare un' altra questione, entro il perimetro degli studi dedicati al segretario fiorentino, su cui si siano registrate divergenze interpretative tanto profonde al punto che si trova chi ritiene che il principato civile condensi addirittura la proposta politica del Principe e chi invece in esso vede un sistema di potere che Machiavelli condanna, auspicandone il rapido trascendimento verso un regime assoluto, per la sua costitutiva fragilità ${ }^{1}$. Forse mai così poche pagine hanno dato luogo a così differenti e opposte letture. E a riprova della difficoltà ad intendersi, almeno nei termini di una convergenza di massima, sul contenuto del capitolo de principatu civili, si considerino anche gli a volte corposi ripensamenti e rimaneggiamenti cui non pochi studiosi hanno sottoposto le loro stesse precedenti interpretazioni, ben oltre la soglia di quello che può accettarsi come fisiologico lavorio critico $^{2}$.

Bisogna ammettere che a provocare questo stato di cose, Machiavelli vi ha messo molto del suo. Il capitolo IX del Principe è, intanto, l'unico luogo dell'intera sua opera in cui ha esplicitamente messo a tema la nozione di principato civile; l'argomentazione che il capitolo, già tra i più brevi del trattato, svolge è estremamente asciutta $\mathrm{e}$ ridotta all'osso; né si distende, se non occasionalmente, a delucidare con dovizia il significato dei concetti evocati; e questo quando va bene, perché in alcuni passaggi, per inseguire un'estrema concentrazione di pensiero, Machiavelli mette a rischio la stessa coerenza del ragionamento. Si aggiunga che l'espressione principato civile, oltre che nel titolo, ricorre una sola volta in tutto il capitolo - e mai più nell'intera opera machiavelliana -, ed anche il lettore più esigente e severo si disporrà con benevola comprensione verso colui che ha deciso, per insondabili motivi, di dedicare il suo tempo a quest'oggetto non a caso sfuggente.

«Teoria politica» XXIV, n. 3, 2008, pp. 61-88 
Proprio la resistenza, per così dire, che il IX capitolo oppone alla sua decifrazione fa sorgere la domanda - meglio, la speranza - sull'eventuale presenza tra gli scritti machiavelliani di qualche altra pagina che analizzi una situazione in qualche modo sovrapponibile a quella del principato civile, pur senza nominarlo. Chiari sarebbero $i$ benefici che l'occorrere di una simile circostanza consentirebbe: il confronto e l'incrocio fra i due testi potrebbe certamente contribuire a rischiarare e a determinare con maggiore precisione l'intendimento che Machiavelli si proponeva nel comporre il capitolo dedicato al principato civile. Orbene, pare di potere dire che un candidato con buone carte per aspirare a questo ruolo ci sia. Si tratta del capitolo XL del primo libro dei Discorsi, in cui viene esaminata la vicenda legata all'istituzione a Roma, nel 451 a. C., del decemvirato e, in particolare, del suo leader Appio Claudio ${ }^{3}$.

Proviamo a fotografare i punti di contatto e le somiglianze tra Principe IX e Discorsi $\mathrm{IXL}^{4}$. In entrambi i capitoli viene tratteggiata la genesi di un potere monarchico entro una cornice repubblicana come conseguenza dell'esasperarsi del contrasto fra i «dua umori» che « in ogni città si truovano », nobili e popolo. Al desiderio del popolo di «non essere comandato né oppresso da' grandi » e dei grandi di «comandare e opprimere el populo» del nono del Principe, fa eco il «desiderio del popolo d'essere libero»e il «troppo desiderio de' nobili di comandare» del capitolo I XL dei Discorsi come potenziale fattore di destabilizzazione delle istituzioni del «vivere libero». E se è vero che in Discorsi I XL Machiavelli attribuisce al popolo un ruolo attivo nel sabotaggio delle istituzioni repubblicane, mentre nel Principe gli assegna unicamente la volontà di sottrarsi alle mire oppressive dei grandi, la sostanza delle cose però non muta, dal momento che è comunque nel contrasto fra $\mathrm{i}$ «dua umori» che va rintracciata, in entrambi i casi, la possibilità che sorga il potere regio.

Sia Principe IX che Discorsi I XL, poi, considerano il contrasto tra popolo e nobili in termini astratti e generali, a prescindere dalle variabili che sul suo svolgimento ed esito il contesto sociale concreto e individuato potrebbe introdurre. Altrove Machiavelli aveva tenuto a distinguere il diverso effetto «dei tumulti e altri scandoli» tra $\mathrm{i}$ «dua umori» sulla base della situazione sociale, rimarcando il loro effetto positivo per la prosperità di una repubblica «dove la materia non è corrotta $»^{5}$, per far discendere da essi conseguenze negative solo nei casi segnati da grave corruzione. Nei testi che stiamo considerando il carattere eversivo nei riguardi delle istituzioni si presenta come un dato, per così dire, originario della dialettica fra nobili e popolo. La degenerazione del conflitto fra le due forze sociali fondamentali che «in ogni città si truovano» ha carattere strutturale, è insito come potenzialità sempre passibile di attualizzazione negli opposti interessi che connotano l'agire dei grandi e del popolo. La differenza, su questo punto, tra Principe IX e Discorsi I XL è solo di metodo: nel primo Machiavelli opta per un'esposizione che si tiene stretta al puro profilo concettuale delle conseguenze del conflitto fra gli «umori», con marginali riferimenti storici, nel secondo è il disteso racconto di una concreta vicenda storica - quella che portò all'istituzione del decemvirato - a fornire la scala per salire alla formulazione di un discorso teorico generale.

Come in Principe IX si ascende al principato civile non con l'attiva azione di conquista del «privato cittadino» che aspira al potere, ma «o con il favore del popu- 
lo o con quello dei grandi», così Appio, nel quale sostanzialmente «si ridusse tutta l'autorità dei suoi compagni» del decemvirato, lo ricevette dai cittadini romani. È vero che ad Appio il potere fu conferito da entrambi gli « umori» e non, come prevede la dottrina del principato civile, dall'uno o dall'altro. Ma il consenso generale con cui furono creati i decemviri fu in realtà il frutto di un compromesso per uscire dallo stallo politico, da cui ciascuna parte però, segretamente, ambiva a trarre un beneficio esclusivo a danno dell'altra. Esattamente come nel capitolo IX del trattato, l'affidarsi ad un'autorità principesca è compiuta sin dall'inizio con intenti opposti e inconciliabili, da parte dei grandi per «sfogare» sotto lo scudo di Appio e dei suoi compagni «il troppo desiderio... di comandare», e da parte del popolo per «il troppo desiderio [...] d'essere libero». D'altronde, è lo stesso Machiavelli a guidarci oltre il velo dell'apparente convergenza delle classi sociali, affermando che la creazione del decemvirato fu la conseguenza dell'incapacità a convenire «a fare una legge in favore della libertà» che spinse «qualcuna delle parti a favorire uno» non per il bene comune ma per il tornaconto particolare.

Ma l'accordo fra il IX del Principe e Discorsi I XL è ancora più profondo e diffuso, riguardando perfino le qualità che sono richieste a chi aspira al potere della propria città quando le istituzioni repubblicane sono messe a rischio dalle lotte sociali. Né tutta virtù né tutta fortuna, dice Machiavelli nel Principe, serve all'aspirante principe, ma piuttosto «una astuzia fortunata» ${ }^{6}$, dove la fortuna indica la sua dipendenza dall'evoluzione di una situazione indisponibile, almeno in larga misura, al suo controllo - senza l'esasperarsi del conflitto sociale i suoi concittadini non sentirebbero la necessità di affidarsi a lui, e la sua ambizione di potere, di conseguenza, non avrebbe l'«occasione» per manifestarsi —, e l'astuzia la capacità di dissimulare le sue vere intenzioni - che non certo sono indirizzate a riformare le istituzioni repubblicane - , e che, qualora conosciute, metterebbero sull'avviso l'uno e l'altro « umore» che non più pertanto gli conferirebbero il proprio favore. Benché non adoperi l'espressione «astuzia fortunata» per caratterizzarne l'ascesa, è difficile contestare che per Machiavelli Appio non rientri tra coloro che hanno costruito il proprio successo su questo binomio. Il «sagace e inquieto » Appio si rivelò, almeno fino ad un certo punto, maestro nell'arte volpina di adoperare l'astuzia: "perché l'astuzia sua nello ingannare la plebe, simulando d'essere uomo popolare, fu bene usata », come recita il supplemento d'indagine che al personaggio dedica il capitolo I XLI dei Discorsi ${ }^{7}$. Ma come in Principe IX, questa sua virtù però nessun frutto gli avrebbe arrecato se $\mathrm{i}$ suoi concittadini, con la loro rivalità, non gli avessero porto l'« occasione » fortunata per farla valere.

Del tutto identica è, inoltre, la linea di condotta che ispirò Appio nella fase di consolidamento del potere rispetto a quella caldeggiata nel capitolo de principatu civili. Chi giunge al potere in conseguenza del conflitto fra grandi e popolo non può di certo sperare di fortificare la propria posizione sul consenso dell'intera comunità politica. È scontato che il sostegno di cui può godere l'aspirante principe è quello della classe il cui interesse è disposto a garantire. L'adozione di una politica volta a far prevalere l'interesse generale su quello di parte procurerebbe al principe solo l'ostilità generale. Visto che ciascuna fazione si attende la tutela del proprio bene 
particolare, il principe non ha alternativa: può cercare e ottenere l'appoggio di una sola classe sociale, soddisfacendone le richieste. Nel nono capitolo Machiavelli spiega che il principe può collocare la propria base politica di consenso o nella nobiltà o nel popolo. In linea di principio nulla osta a che il principe opti per l'una o l'altra strada. Ma poi Machiavelli precisa che il principe giunto al potere col sostegno dei grandi incontra difficoltà di gran lunga superiori a conservarlo rispetto a colui che ha invece il sostegno del popolo. Le ragioni per cui un principe dovrebbe preferire il favore del popolo a quello dei nobili costituiscono uno dei pochi aspetti svolti in modo analiticamente articolato da Principe IX. La convinzione di appartenere ad una classe destinata a comandare, la smodata ambizione di ricchezza e potere, la maggiore intelligenza ed esperienza politica, renderebbero la nobiltà un'insidia permanente per quel principe che fosse stato elevato al potere con il suo favore: i suoi sostenitori, infatti, potrebbero repentinamente mutarsi nei suoi peggiori nemici. Mal disposta a riconoscergli un'autonomia politica effettiva, la nobiltà potrebbe presto presentare al principe un conto salato, disarcionandolo.

Assai più vantaggiosa è invece la posizione del principe che decide di fondare sul popolo. Aspirando unicamente «a non essere oppresso», il popolo ricambierà sempre col proprio sostegno il principe che «ne pigli la protezione», garantendo ordine e sicurezza. Di qui, allora, l'ineludibile esigenza che il principe, nell'eventualità sia asceso al potere col loro favore, si liberi dell'abbraccio dei grandi, prima di esserne soffocato, per cercare il consenso del popolo. E Machiavelli in modo imperativo conclude, nel nono capitolo, che « a uno principe è necessario avere il populo amico, altrimenti non ha nelle avversità remedio». A convalidare questo principio, viene proposto l'esempio dello spartano Nabide che «sostenne la ossidione di tutta Grecia e di uno esercito romano vittoriosissimo, e difese contro a quelli la patria sua e il suo stato; e gli bastò solo, sopravvenendo el periculo, assicurarsi di pochi; che, se gli avessi avuto el populo inimico, questo non li bastava».

Machiavelli accompagna con gli stessi concetti l'esame del comportamento tenuto da Appio nel capitolo I XL dei Discorsi. Un principe che voglia essere tale non può mai interamente fidarsi della nobiltà «per l'ambizione grande e grande avarizia che è in lei ». E anche riconosciuto che il «desiderio»di «tiranneggiare» dei nobili può a volte giovare alla causa del principe, quella parte dei grandi che inevitabilmente rimarrebbe esclusa dal potere gli sarà sempre contro, fonte continua di minaccia per lui. Gravissimo fu quindi, commenta Machiavelli, l'errore di Appio quando lasciò il popolo per accostarsi alla nobiltà, perché si fece nemico di coloro, il popolo, che avevano concorso a dargli il potere, «e che gliele potevano mantenere, e amico di quelli che non erano concorsi a dargliene, e che non gliene arebbono potuto mantenere; e perdessi coloro che gli erano amici, e cercò di avere amici quegli che non gli potevano essere amici», i nobili. Da questo giudizio Machiavelli fa scaturire la medesima regola generale che abbiamo già incontrato e che sembra, anzi, trascritta pari pari dal nono capitolo del Principe: i principi che «hanno amico l'universale e inimici i grandi, sono più sicuri, per essere la loro violenza sostenuta da maggiori forze che quella di coloro che hanno per inimico il popolo e amica la nobiltà». Chi può allora sorprendersi del fatto che, per illustrare questo fondamentale principio di 
prudenza politica, Machiavelli non trovi diverso esempio di quello dello spartano Nabide già adoperato in Principe IX? Solo i sovrani che godono del sostegno popolare si mantengono facilmente, «perché, con quello favore, bastano a conservarli le forze intrinseche, come bastarono a Nabide, tiranno di Sparta, quando tutta la Grecia e il popolo romano lo assaltò: il quale assicuratosi di pochi nobili, avendo amico il popolo, con quello si difese, il che non arebbe potuto fare avendolo inimico».

La lettura e il raffronto fra Principe IX e Discorsi I XL rende irresistibile la tentazione di riunire i due testi sotto lo stesso comune denominatore. La loro convergenza appare, infatti, strutturale e non circoscritta ad aspetti secondari o di superficie. Analoga è la situazione politica esaminata, analoga l'analisi, analoghe le conclusioni che Machiavelli ricava. Si potrebbe tuttavia osservare che la vicenda di Appio e dei decemviri esposta in Discorsi I XL si discosti dal quadro tracciato in Principe IX su due punti di rilievo che, spezzando la corrispondenza, vanificherebbero la possibilità di un confronto fra i due capitoli. Il primo concerne il fatto che mentre nel Principe il conflitto fra $\mathrm{i}$ «dua umori » porta al conferimento ad un privato di un potere esercitato al di fuori dell'ordinamento costituzionale, nei Discorsi, invece, Appio si trova alla testa di un'istituzione della repubblica alla quale, inoltre, è giunto per la via prevista dalla legge. Appio, insomma, non è un uomo che si è fatto strada fuori delle istituzioni, finendo per imporsi ad esse, ma detiene un potere legittimo, ottenuto tramite le normali procedure costituzionali; il secondo punto riguarda la definizione di tiranno che Machiavelli riserva ad Appio, definizione che sembrerebbe collocarne l'esperienza entro un universo di significati totalmente estraneo a quello del principato oggetto del capitolo IX. Cosa avrebbero, verrebbe infatti da chiedersi, in comune la tirannide dei dieci e di Appio e il principe civile?

Per fortuna, almeno per rispondere alla prima osservazione, non è richiesto un grande sforzo interpretativo, perché è sufficiente dare la parola direttamente a Machiavelli che, nei capitoli immediatamente precedenti il XL del primo libro, si era a sua volta preoccupato della questione, scrivendo: «E' pare contrario a quel che di sopra è discorso (che quella autorità che si occupa con violenza, non quella che è data con gli suffragii, nuoce alle republiche) la elezione de' dieci cittadini creati dal popolo romano per fare le leggi di Roma: i quali ne diventarono con il tempo tiranni, e sanza alcun rispetto occuparono la libertà di quella. Dove si debbe considerare i modi del dare l'autorità e il tempo per che la si dà. E quando e' si dia autorità libera sempre fia pericolosa, e farà gli effetti o buoni o rei secondo che fiano rei o buoni coloro a chi la sarà data ${ }^{8}$. Queste parole sono dettate a Machiavelli dall'analisi che aveva condotto nei capitoli XXXIII e XXXIV. Nel primo aveva considerato quali misure debba adottare una repubblica per contrastare quei casi in cui «si lascia pigliare a uno cittadino più forze che non è ragionevole ${ }^{9}$. Che una repubblica debba far di tutto per evitare che un privato acquisti una posizione di preminenza nella vita pubblica è spiegato col fatto che, approfittando dell'inevitabile favore dei suoi cittadini, «se in quello è punto di ambizione ${ }^{10} \mathrm{~s}^{\prime}$ impossesserà del potere. Non si fatica a riconoscere in queste parole una tipica situazione da principato civile, come, peraltro, l'esempio di Cosimo de' Medici che viene allegato conferma: «Cosimo de' Medici [...] venne 
in tanta riputazione col favore che gli dette la sua prudenza e ignoranza degli altri cittadini, che ei cominciò a far paura allo stato ${ }^{11}$. Nel capitolo che segue, il XXXIV, Machiavelli aveva invece opposto alle «forze» acquisite da un privato cittadino quelle che sono costituite « secondo gli ordini publici» ${ }^{12}$. Queste, come dimostra l'istituzione dittatoria della repubblica romana, fecero « sempre bene alla città » ${ }^{13}$, esercitandosi il loro potere entro i confini posti dalla costituzione e dalle altre magistrature. Il dittatore, infatti, disponeva sì di un notevole potere, «ma non poteva fare cosa che fussi in diminuzione dello stato, come sarebbe stato tôrre autorità al senato o al popolo, disfare gli ordini vecchi della città e farne de' nuovi ${ }^{14}$.

Nell'introdurne la vicenda, nel capitolo I XXXV, che come ormai sappiamo riceverà una trattazione sistematica in I XL, Machiavelli si sente in dovere di spiegare al lettore perché il caso di Appio e del decemvirato debba ascriversi alla tipologia dei poteri esaminata nel capitolo I XXXIII. A tutta prima parrebbe ovvio invece equiparare il decemvirato alla dittatura, per essere il suo potere «dato secondo gli ordini publici». Ma se si considera, chiarisce Machiavelli, che i decemviri ottennero un potere assoluto, non arginato e delimitato dalle altre magistrature, e per un lungo periodo di tempo per giunta, allora la loro posizione deve essere ricondotta a quella propria del privato cittadino che, al di fuori delle istituzioni, piglia « più forze che non è ragionevole ». Al di là delle differenze formali, è Machiavelli stesso, dunque, a indicare la convergenza sostanziale fra il caso di Appio e dei decemviri e quello del principato civile affrontato nel nono del Principe. Nella valutazione di Machiavelli, l'elemento decisivo è costituito dal fatto che il conflitto fra grandi e popolo può portare all'assegnazione di un potere tale, poco importa se per via privata o pubblica, da imporsi, esautorandole, alle istituzioni repubblicane e forzare il passaggio al principato.

E veniamo al secondo punto, che riguarda la definizione di tirannide con cui Machiavelli designa il potere politico di Appio e dei dieci. Come detto, essa sembrerebbe a tutta prima senz' altro rinviare ad un ambito concettuale estraneo a quello del principato civile del capitolo IX, cosa che, se così effettivamente fosse, sarebbe ovviamente assai grave, perché comprometterebbe alla radice l'impianto stesso del presente lavoro, palesando l'irriducibilità delle situazioni svolte nei testi del Principe e dei Discorsi considerati. Si deve allora, in via preliminare, precisare il significato che Machiavelli attribuisce al concetto di tirannide in I XL.

La dialettica politica fra i due umori delineata nel capitolo I XL restringe l'alternativa delle forme di governo alla repubblica e alla tirannide. Machiavelli esclude, o quantomeno non considera in questo luogo, altro possibile esito. Questa caratterizzazione dello sbocco politico dello scontro sociale segnala forse che in Machiavelli si sia verificato un movimento di pensiero che lo ha portato a rinnegare la prospettiva del trattato per la convinzione, maturata solo nei Discorsi, che ogni reggimento monocratico sia nella sostanza un regime oppressivo, appunto tirannico ${ }^{15}$ ? All'apprezzamento per il principe che nel capitolo IX del Principe sottrae la compagine sociale alla sua deriva politica, subentrerebbe, quindi, nei Discorsi l'idea che, a petto della repubblica, si erga solamente la tirannide, il regime della violenza e dell'abuso del potere, che riduce a sé l'intero spettro delle forme monocratiche? Difficile crederlo. Non è, infatti, arduo mostrare come la distinzione fra monarchia e tirannide sia tutt'altro che 
sconosciuta al Machiavelli dei Discorsi ${ }^{16}$. Si guardi solamente al capitolo II del primo libro. Riproponendo, adattata alle proprie esigenze, la teoria polibiana dell' anacyclo$\operatorname{sis}^{17}$, Machiavelli distingue con cura la forma buona del regime monarchico dalla sua degenerazione tirannica: non appena il metodo elettivo fu sostituito dalla successione ereditaria, «subito cominciarono gli eredi a degenerare dai loro antichi, e lasciando l'opere virtuose pensavano che i principi non avessero a fare altro che superare gli altri di suntuosità e di lascivia e d'ogni altra qualità di licenza: in modo che, cominciando il principe a essere odiato e per tale odio a temere, e passando tosto dal timore all'offesa, ne nasceva presto una tirannide ${ }^{18}$.

Nel brutale e arbitrario uso del potere contro il bene comune e a favore dell'interesse particolare di chi comanda, Machiavelli dunque situa anche nei Discorsi l'insuperabile discrimine, così carico di echi classici, tra la tirannide e la monarchia. Non deve tuttavia sorprendere, in un pensatore quale il segretario fiorentino, poco incline al rigore sistematico, che la medesima espressione formale non denoti sempre la medesima sostanza concettuale. È tutt'altro che infrequente, infatti, nelle pagine dei Discorsi imbattersi nel nome della tirannide quale specificazione di un genere più comprensivo, quello della «potestà assoluta». Quando ciò accade Machiavelli tende ad usare indifferentemente tirannide, regno e principato parendogli che tutt'e tre possano esprimere adeguatamente la realtà concettuale dell'assolutezza del potere. La cosa risulta evidente quando si leggano in successione i capitoli XXV e XXVI del primo libro dei Discorsi. In Discorsi I XXV, dopo avere constatato che un riformatore deve, nella sua azione di riordinamento di uno stato, sempre «ritenere l'ombra almanco de' modi antichi, acciò che a' popoli non paia avere mutato ordine ${ }^{19}$, Machiavelli così conclude il capitolo: «e questo, come ho detto, debbe osservare colui che vuole ordinare uno vivere politico, o per via di repubblica o di regno; ma quello che vuole fare una potestà assoluta, la quale dagli autori è chiamata tirannide, debbe rinnovare ogni cosa, come nel seguente capitolo si dirà ${ }^{20}$. Il testo introduce un nuovo criterio di partizione, rispetto a quello che ricalca Polibio del secondo capitolo del primo libro, dei regimi politici. Questi ora si riducono a due specie: quelli conformi al «vivere politico » 0 , come più frequentemente usa Machiavelli, al «vivere civile» e quelli «assoluti ${ }^{21}$. La distinzione rimanda al modo in cui il potere è organizzato: «civili » sono quei governi in cui il potere si articola in «ordini» e «magistrature» secondo competenze, modi e limiti fissati da una costituzione; « assoluti » sono invece i governi in cui tutto il potere si concentra in una sola volontà, non importa se di un uomo o di un gruppo, che lo esercita senza limiti, restrizioni e controlli di sorta. In base a tale partizione, Machiavelli raccoglie repubbliche e regni sotto il titolo di governi « civili », mentre assegna la tirannide alla sfera della «potestà assoluta».

Sulla scorta del capitolo I XXV netta sembrerebbe la distinzione tra regno e tirannide, con il primo che rientrerebbe nelle forme politiche «civili» e la seconda in quelle «assolute». Ma quando si ha a che fare con Machiavelli è difficile che le cose filino sempre lisce. Ed in effetti, non appena si volta pagina e si abborda il capitolo XXVI, in cui era stato preso l'impegno di discutere della "potestà assoluta, la quale dagli autori è chiamata tirannide », ci si accorge che non tutto è così netto e chiaro come sembrava un momento prima. Con una certa sorpresa, infatti, si scopre che pro- 
prio il termine tirannide non compare nemmeno una volta, in cui luogo stanno, titolo compreso - «uno principe nuovo, in una città o provincia presa da lui, debbe fare ogni cosa nuova ${ }^{22}-$ una sequenza di «principe», «principato »e « re ${ }^{2}$. L' ${ }^{\prime}$ incipit del capitolo è quanto mai significativo: « qualunque diventa principe o d'una città o d'uno stato (e tanto più quando i fondamenti suoi fussono deboli), e non si volga o per via di regno o di republica alla vita civile, il migliore rimedio che egli abbia a tenere quel principato è, sendo egli nuovo principe, fare ogni cosa in quello stato di nuovo ${ }^{23}$. Certo, la prima parte del brano riconferma l'appartenenza del regno, insieme alla repubblica, alla forma politica del «vivere civile», in opposizione alla forma politica della «potestà assoluta»; ma la sua seconda parte, poi, anziché tirannide, come ci si aspetterebbe, usa per designare la «potestà assoluta» il termine principato, e definisce «nuovo principe» quello che tutto lasciava pensare che sarebbe stato chiamato tiranno. Anche gli esempi che vengono addotti confermano l'identità di significato che i termini tiranno e re, o principe, possiedono quando il concetto inteso è la «potestà assoluta». Il biblico Davide e Filippo di Macedonia, che pure agirono in conformità con quanto era stato indicato in precedenza come comportamento tirannico, avendo disfatto i vecchi ordinamenti e fatti in loro luogo «nuovi governi con nuovi nomi, con nuova autorità, con nuovi uomini » sono chiamati «re ${ }^{24}$. Come pure, nella chiusa del capitolo, è di nuovo il termine «re» che viene adoperato per indicare quei «modi crudelissimi e nemici d'ogni vivere non solamente cristiano, ma umano ${ }^{25}$ che caratterizzano nel momento della sua fondazione la «potestà assoluta».

I capitoli I XXV e XXVI dei Discorsi forniscono gli esempi tra più efficaci della polivalenza semantica del lessico machiavelliano. Regno e principato vengono impiegati sia per riferirsi ai regimi politici «civili» sia in relazione a quelli «assoluti» . È chiaro però che, pur designando un potere che si esprime sempre nella forma monocratica, il significato che assumono varia profondamente: un regno «civile» è ben altra cosa rispetto a un regno «assoluto ». Tuttavia, mettendo da parte tutte le possibili conseguenze che potrebbero derivare da quanto si è detto per l'interpretazione complessiva del suo pensiero, quello che ai fini del nostro discorso preme segnalare è che quando Machiavelli ha in mente la «potestà assoluta» adopera quali sinonimi «regno», «principato» e «tirannide ${ }^{26}$. Questo modo di procedere richiede grande prudenza e attenzione al lettore, per evitare facili fraintendimenti e affrettate conclusioni. Per determinare il significato di regno, ad esempio, occorrerà prima accertarsi, attraverso il contesto, se esso si riferisca a un regime «civile» o a uno «assoluto »; e nei casi in cui il contesto autorizza ad intendere regno quale «potestà assoluta», allora si può fondatamente assimilarne il significato a quello di tirannide, e non ci si dovrà sorprendere se la medesima realtà concettuale venga definita in una pagina in un modo e in un'altra diversamente. Va peraltro aggiunto, a completamento di quanto detto, che la distinzione tra governi «civili» e «assoluti» comporta un'importante ricaduta sul significato del concetto di tirannide che, in quanto «potestà assoluta », tende a cedere parte della connotazione negativa che abitualmente l'accompagna, e in cui va ravvisato un ulteriore elemento che consente di accostare fra di loro regno, principato e tirannide. Nel momento in cui mette l'accento sul carattere assoluto del potere, cioè sulla sua interna strutturazione, Machiavelli fa scivolare in secondo 
piano la destinazione e i fini per cui viene esercitato. Da ciò discende che la «potestà assoluta» non si configura di per sé come una forma politica in cui il potere venga usato contro l'interesse della comunità. Un potere assoluto può, infatti, essere impiegato sia a vantaggio sia contro l'interesse generale, sia con moderazione sia con arbitrio smodato. E che si opti per l'uno o l'altro orientamento dipende unicamente dalla «virtù» di chi concretamente detiene il potere. Non deve allora meravigliare che Machiavelli quando pensa alla «potestà assoluta» sfumi la connotazione negativa del concetto di tirannide, avvicinandone il significato, fino addirittura a sovrapporlo, a quello di regno e di principato ${ }^{27}$.

Può essere utile fornire qualche ulteriore esempio del modo d'impiego da parte di Machiavelli del lessico che concerne le forme del potere monocratico. Dando corpo ad un'annotazione avanzata in I II, il capitolo IX del libro primo dei Discorsi, egli spiega per quale motivo l'opera di Romolo vada annoverata tra quelle dei fondatori degli stati «più conformi a uno vivere civile e libero, che a uno assoluto e tirannico » ${ }^{28}$. Sin dall'inizio Romolo agì in vista del bene comune per « avere quello sùbito ordinato uno senato con il quale si consigliasse, e secondo la oppinione del quale deliberasse », riservando per sé alcun'altra autorità «che comandare agli eserciti » ${ }^{29}$. Romolo si presenta come il fondatore di un regno che rientra nelle forme del «vivere civile», perché ha rinunciato all'istituzione di una «potestà assoluta» che, invece, avrebbe reso il suo titolo di re equivalente a quello di tiranno. Costituendo un senato col quale condivise il potere, il suo regno deve essere incluso nelle forme del «vivere civile». Pure all'ambito del «vivere civile» appartengono regno e re quando Machiavelli li riferisce alla monarchia di Francia, perché «chi ordinò quello stato volle che quegli re dell'armi e del danaio facessero a loro modo, ma che d'ogni altra cosa non ne potessero altrimenti disporre che le leggi si ordinassero ${ }^{30}$. Anche in questo caso, il lettore non può confondere regno e tirannide in quanto la regolazione e la limitazione del potere mediante le leggi e gli ordinamenti impedisce che il regno di Francia, con il suo re, sia una «potestà assoluta ${ }^{31}$.

Quando invece il discorso concerne proprio la «potestà assoluta », allora, i termini di re e principe che Machiavelli impiega vanno presi nello stesso significato di tiranno. Si prenda il capitolo II del secondo libro dei Discorsi: «e veramente maravigliosa cosa è a considerare a quanta grandezza venne Atene per spazio di cento anni, poi che la si liberò dalla tirannide di Pisistrato. Ma sopra tutto maravigliosamente è da considerare a quanta grandezza venne Roma, poi che la si liberò da' suoi re ${ }^{32}$. I re di Roma cui Machiavelli qui si riferisce sono i Tarquini che, alterando gli equilibri costituzionali promossi da Romolo, governarono in modo assolutistico. Essi in nulla si distinguono da Pisistrato: definirli re piuttosto che tiranni non sposta i termini della questione, accomunandoli la sostanza del potere di cui disposero, l'unico aspetto che sembra veramente interessare in questo luogo Machiavelli. Altra prova di come Machiavelli non distingua tra principato e tirannide, quando si occupa della «potestà assoluta », si ricava dall'esame comparato dei capitoli I XVI e III III. Il medesimo problema - le misure da adottare in caso di mutazione di stato - affrontato dai due capitoli viene formulato con una sola, ma significativa variante. Nel capitolo I XVI Machiavelli scrive: «e chi prende a governare una moltitudine, o per via di libertà o per via di 
principato, e non si assicura di coloro che a quello ordine nuovo sono inimici, fa uno stato di poca vita ${ }^{33}$; mentre in III III, ribadendo lo stesso precetto, egli sostituisce solamente il termine tirannide a principato: «e sempre si conoscerà questo per coloro che le cose antiche leggeranno: come dopo una mutazione di stato o da republica in tirannide o da tirannide in republica, è necessaria una esecuzione memorabile contro a' nimici delle condizioni presenti. E chi piglia una tirannide e non ammazza Bruto, e chi fa uno stato libero e non ammazza i figliuoli di Bruto, si mantiene poco tempo $»^{34}$. Nel passaggio da un testo all'altro, il cambiamento terminologico non incide minimamente sul piano semantico. Principato e tirannide si presentano come sinonimi, indicando in entrambe le situazioni il carattere assoluto del potere.

Ma l'esempio più efficace che mostra come la distanza tra principato e tirannide si annulli, allorché l'oggetto della riflessione è la «potestà assoluta» si trova proprio nei capitoli di cui si occupa il presente lavoro. Esso è dato dalla figura di Nabide, che compare sia in Principe IX sia in Discorsi I XL: in entrambi i capitoli Nabide è recato come esempio positivo da imitare; in entrambi i capitoli la sua esemplarità è dovuta alla stessa ragione: avere conquistato il favore popolare; ma nel capitolo IX del Principe è ricordato come «principe delli Spartani», mentre in Discorsi I XL è definito «tiranno di Sparta». Il Nabide «principe» del capitolo IX è certamente la stessa persona, politicamente s'intende, del Nabide «tiranno» del capitolo I XL. Se così non fosse, Machiavelli non avrebbe potuto assegnargli in entrambi i capitoli gli stessi predicati e presentarlo come positivo modello da seguire. Non fa pertanto alcuna differenza che Machiavelli si rivolga a Nabide con l'appellativo di principe o di tiranno, perché nel suo caso la divergenza nominale rimanda ad una più profonda convergenza semantica che si realizza intorno al concetto di «potestà assoluta».

Non si può, dunque, considerare un ostacolo all'identificazione delle situazioni svolte in Principe IX e Discorsi I XL il fatto che nel primo si parli di principato e nel secondo di tirannide. Se si ha presente, poi, che nell'ultimo capoverso del nono capitolo Machiavelli incita i principi a «salire dall'ordine civile allo assoluto », liberandosi dell'intralcio che potrebbe venire loro dalla sopravvivenza delle magistrature repubblicane, che renderebbero «più debole e più pericoloso lo stare loro», perché mai si dovrebbe respingerne il gemellaggio con Discorsi I XL $^{35}$ ? Chi non si lascia ingannare dai riflessi di superficie riconoscerà, dietro la differenza terminologica, la comune natura assoluta del potere presente nei due capitoli ${ }^{36}$. D'altra parte, qualora l'«autorità assoluta» di cui si trovò a beneficiare il tiranno Appio, quando divenne «al tutto principe di Roma», non equivalesse all'ordine assoluto cui deve affrettarsi a «salire» il principe del IX capitolo, come si potrebbe spiegare l'identico precetto che in entrambi i casi Machiavelli impone di seguire al novello sovrano che ha come problema prioritario di «fermare» il proprio potere: ottenere l'amicizia del popolo? Né si può obiettare che, ottenendo l'amicizia del popolo, si sarebbe mutato lo status politico di Appio, che da tirannico sarebbe diventato allora principesco. Il testo del capitolo I XL non offre margini per questa lettura, dal momento che al suo interno Machiavelli utilizza sistematicamente sempre il termine tiranno per indicare la posizione di coloro che si sono procurati l'amicizia dell' « universale ». E ciò perché, quando in gioco è l'assolutezza del potere, tiranno vale principe e viceversa, e non fa differenza 
il ricorso all'uno piuttosto che all'altro termine, essendo la loro sostanza concettuale sempre la medesima. La nozione di «potestà assoluta» va, dunque, riconosciuta come il medio che consente di congiungere il principe, partito civile ma diventato assoluto, del nono capitolo del trattato con la vicenda dei decemviri capeggiati da Appio Claudio di Discorsi I XL che disposero dell' « autorità assoluta » a Roma. Non è allora, crediamo, esercizio di fantasia immaginare che, se il tiranno Appio avesse mantenuta l'amicizia del popolo, il suo nome avrebbe potuto fare capolino, proprio accanto a quello di Nabide, nel IX del Principe, come esempio da seguire per chi, arrivato al potere per il conflitto fra gli «umori», aveva urgenza di consolidare il proprio potere.

Verificata la stretta corrispondenza tra il tema e lo svolgimento di Principe IX e di Discorsi I XL, la questione che vorremmo quindi sollevare riguarda l'ausilio che può venire dal secondo per precisare l'intento con cui Machiavelli compose il primo e per determinarne con esattezza la posizione nel suo sistema complessivo di pensiero. In particolare, sono due gli aspetti che interessa qui chiarire: 1) la possibilità di intendere la teorizzazione del principato filopopolare, come a molti è parso ${ }^{37}$, quale soluzione istituzionale che Machiavelli era andato elaborando per risolvere la crisi di una repubblica; 2) il senso da attribuire al precetto di farsi amico il popolo che viene indirizzato al principe. Perché il capitolo I XL dei Discorsi possa essere d'aiuto in questo compito, si richiede che esso contenga almeno qualche informazione supplementare rispetto a quelle presenti nel capitolo de principatu civili. Da questo punto di vista non si può certamente dire che deluda le attese, presentando un elemento di assoluta novità nella doppia prospettiva in cui considera il conflitto politico entro la cornice istituzionale repubblicana da cui origina il principato. Questo è esaminato nella sua genesi sia dalla prospettiva delle classi sociali, nobili e popolo, sia da quella di chi, dal loro contrasto, viene condotto al potere. Nelle battute introduttive del capitolo, Machiavelli dichiara di essere stato attratto dalla vicenda del decemvirato per gli insegnamenti che esso offre «così per coloro che vogliono mantenere una republica libera, come per quelli che disegnassono sottometterla»; in quanto, aggiunge, a scrutarne a fondo il corso «si vedrà molti errori fatti dal senato e dalla plebe in disfavore della libertà, e molti errori fatti da Appio, capo del Decemvirato, in disfavore di quella tirannide ch'egli si aveva presupposto stabilire in Roma». Nel IX capitolo del trattato, Machiavelli aveva ristretto il suo angolo visuale alla sola considerazione dei passi da fare da parte del principe giunto al potere «col favore del populo o con quello de' grandi » in vista della stabilizzazione della sua posizione, in Discorsi I XL, invece, adotta un'ottica più comprensiva, passando al setaccio della sua analisi sia il comportamento delle parti sociali sia quello dell'aspirante signore. La prospettiva allargata di Discorsi I $\mathrm{XL}$, delineando l'immagine integrale del conflitto politico e portando in primo piano le istanze degli «umori» sociali, che prima erano rimaste sullo sfondo, svolge un motivo che era stato lasciato in ombra da Principe IX. Nel Principe Machiavelli non aveva avuto occhi che per i problemi di fondazione del potere del nuovo sovrano; gli interessi delle classi sociali non rientravano nel raggio della sua attenzione. In Discorsi I XL invece la sua analisi si appunta anche sulle scelte operate dal popolo e dai nobili non meno che su quelle operate dal principe. C'è allora da domandarsi se 
proprio le considerazioni di Machiavelli sugli « umori » non finiscano per illuminare di nuova luce e per collocare entro nuove relazioni la dottrina del principato tratteggiata nel capitolo IX, rivelando insospettati significati che impongano, infine, di ripensare l'opinione che di esso ci si era formati. Urge ripercorrere con Machiavelli proprio quella parte che Discorsi I XL svolge in esclusiva, vale a dire i «molti errori fatti dal senato e dalla plebe in disfavore della libertà », per stabilire quali riflessi possono venirne per la determinazione del significato dell'operazione condotta nel capitolo dedicato al principato civile.

L'analisi che Machiavelli svolge colloca sotto il segno dell' «errore» il comportamento del popolo e dei nobili. L' «errore» che viene imputato agli «umori» non riguarda il fine che si proponevano di conseguire, ma i mezzi che utilizzarono. Che l'intento del popolo e dei grandi fosse quello di riformare le istituzioni repubblicane e che, a tale fine, fosse inviato «Spurio Postumio con duoi altri cittadini a Atene, per gli esempi di quelle leggi che Solone dette a quella città, acciò che sopra quelle potessono fondare le leggi romane», merita il deciso apprezzamento di Machiavelli. Nel corso della sua disamina egli mostra di considerare un presupposto che nemmeno merita di essere discusso quello per cui l'interesse politico delle classi sociali dovesse portare a che «si stabilisse più la libertà di quello stato », rinnovando e rafforzando, quindi, la repubblica. Per quanto aspro si fosse fatto lo scontro sociale e per quanto ciascuna classe ambisse ad imporre all'altra la propria esclusiva volontà, Machiavelli sembra ritenere che la logica politica non potesse che spingere gli «umori» a permanere all'interno del perimetro delle istituzioni repubblicane ${ }^{38}$. Ma proprio perché non era certo nei propositi di alcuno preparare la transizione ad un regime di tipo monarchico, proprio perché non si era affacciata la considerazione che lo scudo monarchico potesse meglio tutelare gli interessi particolari degli uni dalla volontà prevaricatrice degli altri, Machiavelli qualifica poi come «errore» gravissimo il modo di procedere del popolo e dei nobili, che credettero di potere conseguire la riforma delle istituzioni repubblicane concentrando l'intero potere in una sola magistratura. Facendo infatti «al tutto principe di Roma» «dieci cittadini per uno anno, intra' quali fu creato Appio Claudio, uomo sagace e inquieto», e togliendo, quindi, «tutti gli altri magistrati, e in particolare i tribuni e i consoli », non si resero conto di preparare non la riforma della repubblica, ma l'avvento di un potere assoluto che nessuno, se non l'aspirante principe, mostrava di volere. Sempre accade che, quando ci si immette su questa via, commenta Machiavelli, «subito la tirannide surge», con la repubblica che cede alla «potestà assoluta». Ben altro modo di procedere si sarebbe dovuto seguire. Benché un' «autorità assoluta» possa rendersi necessaria laddove è richiesto un intervento costituente, «il popolo debbe, quando egli ordina i magistrati, fargli in modo che gli abbino avere qualche rispetto a diventare scellerati ». Il che vuol dire conferire loro un potere per un breve tempo e, comunque, ponendo «loro guardia per mantenergli buoni ». Occorreva in altri termini, sebbene Machiavelli non lo dica esplicitamente, battere la strada che si seguiva usualmente a Roma nella creazione della dittatura che, sia per la sua durata limitata sia perché non poteva annullare le istituzioni dell'ordinamento costituzionale, fece sempre bene e non fu mai la leva per impiantare un potere personale e assoluto. Fecero, pertanto, «il senato e il popolo in questa creazione del 
Decemvirato errori grandissimi», e solo per gli «errori » altrettanto gravi commessi da Appio fu possibile riacquistare infine quella libertà politica che sembrava irrimediabilmente perduta. Ché se Appio fosse stato accorto, come pure inizialmente fu, e si fosse mantenuto il popolo amico, allora il destino della repubblica sarebbe stato segnato e «la sua tirannide arebbe presa più vita, e non sarebbe mancata sì presto ».

Nella valutazione del loro comportamento, Machiavelli imputa gli «errori grandissimi » commessi dal popolo e dai grandi alla perdita della lucidità politica, all'incapacità di calcolare con fredda razionalità i propri interessi autentici a causa del prevalere delle passioni accecanti. Il «troppo desiderio del popolo d'essere libero » e il « troppo desiderio de' nobili di comandare » impedirono ai « dua umori» di riconoscere che solo la via dell'accordo, nella reciproca rinuncia a parte delle proprie pretese, poteva portare «a fare una legge in favore della libertà ». L'odio e la diffidenza che correvano tra nobili e popolo, rendendo impraticabile un'intesa, li illusero che si potesse uscire dallo stallo in cui si erano cacciati gettandosi «qualcuna delle parti a favorire uno ». Il timore di ciascuna classe di soccombere all'altra in una trattativa diretta suggerì di fare ciò che la lucida analisi razionale sarebbe tornata sempre a sconsigliare: creare un potere assoluto che arbitrasse imparzialmente il conflitto sociale. Ma come si può essere così ingenui da pensare, annota Machiavelli, che chi si trovi a disporre di un sì grande potere, e « sia savio », non lo usi poi per sé e non per la riforma delle istituzioni del « vivere libero »? Non c'è proprio da sorprendersi allora che, facendosi guidare ciascuna delle parti dal «desiderio [...] l'una di spegnere il nome consolare, l'altra il tribunizio », si aprisse a Roma la strada alla tirannide, piuttosto che al rinnovamento della repubblica, con l'arbitro che facendosi tiranno impose la spietata legge della servitù all'intero corpo sociale, che se ne accorse solo quando non aveva più « dove rifuggire».

Il dato che più colpisce dell'analisi delle scelte degli « umori » condotta in Discorsi I XL, è sicuramente costituito dalla netta condanna che viene fatta cadere sulla testa delle classi sociali, per avere con il loro comportamento sconsiderato rischiato di provocare la fine della repubblica e l'avvento di un regime monocratico. Nel giudizio di Machiavelli è contenuta la convinzione che, per quanto malconcia, la repubblica offra sempre la cornice istituzionale più adatta a rispondere alle aspirazioni politiche del popolo e di grandi. Né gli sembra che la perdita della libertà politica possa tradursi in un guadagno, nemmeno per quella parte i cui interessi il principe prendesse eventualmente a difendere in esclusiva contro l'altra. Ad osservare, quindi, il corso politico dalla prospettiva delle classi sociali, la repubblica rappresenta un valore assoluto che non deve essere mai sacrificato. E ciò, come risulta dal carattere di esemplarità che attribuisce alla vicenda dell' istituzione del decemvirato, si configura per Machiavelli come un principio generale valido per tutte le situazioni. In nessun passaggio del capitolo, s'affaccia infatti l'idea che il principio possa prevedere eccezioni: non sono segnalate circostanze, neanche per casi di estremo deterioramento della vita civile e politica, in cui possa non essere un «errore» per le classi sociali abbandonare la repubblica. La nettezza del giudizio negativo sull'operato degli «umori» non lascia spazio per ritenere giustificabile la transizione al principato nemmeno laddove quest'ultimo possa restituire alla compagine politica ordine e sicurezza compromessi da una conflittualità sociale fattasi incandescente. 
A rimanere allora a Discorsi I XL, sembra proprio che Machiavelli consideri lo sbocco regio la principale insidia che minaccia una comunità organizzata nella forma istituzionale della repubblica. L'esigenza che ciascuna classe moderi le proprie pretese trova la sua ragion d'essere nell'esca che altrimenti si fornirebbe a chi vuole fare una «potestà assoluta». Quest'ultima, pertanto, più che essere la risposta al malessere di una repubblica, si configura come l'emblema di una realtà politica che ha toccato il suo estremo decadimento e che non ha più energie per risollevarsi. Lungi, dall'essere l'inizio di un cammino di risalita, l'approdo al principato è il fondo di un precipizio dove non giunge più luce ${ }^{39}$.

Si può capire l'effetto spiazzante che, a prima vista, questa posizione può determinare in chi vi acceda direttamente dal capitolo IX del trattato, dove la prospettiva dell'analisi era costituita dalle sole esigenze del principe. Spontaneo verrebbe da concludere che un terremoto deve essersi in qualche punto verificato nel tempo tra la stesura del trattato e la composizione del commento liviano nell'universo concettuale di Machiavelli. Per quanto possano esserne indecifrabili le ragioni, non parrebbe negabile che un sommovimento di prima grandezza deve avere provocato il capovolgimento dell'impostazione del IX capitolo del Principe, se in Discorsi I XL si legge che il regime di uno solo coincide con l'asservimento dell'intera comunità. Le pagine del de principatu civili parevano assicurare che nella stretta alleanza tra principe e popolo era da individuarsi la positiva soluzione per una repubblica in caduta libera, e che il popolo aveva più che buoni motivi, e nessuna controindicazione, per mettersi sotto la protezione di un principe. Il severo e inappellabile giudizio di condanna degli «umori» sociali che, per non avere tenuto a freno la loro smodata ambizione, si sono lasciati tentare dalla prospettiva regia fa di Discorsi I XL una scolorina teorica che inesorabilmente copre di una mano di bianco la posizione espressa in Principe IX.

Ma si è davvero autorizzati a concludere così? Si deve assumere che Discorsi I XL segni l'accantonamento di quanto era stato teorizzato in Principe IX e che i due capitoli siano, quindi, l'emblema di due diverse stagioni della riflessione di Machiavelli, in cui si passerebbe da una visione salvifica del principato ad una demonizzante? È lecito pensare che quando scrive i Discorsi Machiavelli abbia ripudiato la dottrina del principato popolare, su cui sembrava avere puntato tutto in precedenza? La risposta a queste domande sarebbe senz'altro affermativa, se Discorsi I XL non riproponesse poi, accanto al perentorio comando a popolo e grandi a non uscire dal confine repubblicano, la stessa ricetta di Principe IX quando l'attenzione cade invece sul comportamento che deve osservare l'aspirante signore nella fondazione del suo potere. La regola politica per cui, a conservare il potere, occorre «che sia più potente chi sforza che chi è sforzato » porta Machiavelli a tenersi saldo anche in Discorsi I XL alla convinzione che per un principe l'amicizia del popolo sia sempre essenziale. Di qui proprio Machiavelli fa discendere, come si è già avuto modo di dire, il positivo giudizio sulle scelte iniziali di Appio che opportunamente si accostò al popolo, reprimendo in sé la tendenza a collocarsi dalla parte della nobiltà, cui lo inclinava la sua origine sociale. E se egli avesse insistito in questa linea di condotta avrebbe fatto esattamente quanto necessitava perché «quella sua tirannide» prendesse «più vita»e non mancasse «sì presto ». Per non avere mantenuto l'indirizzo filopopolare, Machia- 
velli colloca anche le scelte di Appio sotto il segno dell' «errore »: più grande «errore » non avrebbe potuto commettere nell'opera di consolidamento della sua tirannide di quello di allontanarsi dal popolo e di avvicinarsi ai grandi, perché si fece inimico di coloro «che gliele potevano mantenere » e amico di coloro «che non gliele arebbono potuta mantenere». Ma questo giudizio sull'operato di Appio, con la sottolineatura degli «errori» che condussero al naufragio del suo progetto politico, rende fin troppo evidente che Machiavelli non si sia allontanato dalla temperie concettuale del capitolo IX del Principe, e che esso continui ad ispirarne la riflessione quando il suo oggetto sia costituito dalle condizioni che rendono possibile il potere regio. Non si può nutrire allora alcun dubbio sulla permanente validità per Machiavelli, anche nel capitolo I XL dei Discorsi, delle indicazioni fornite al principe preoccupato di stabilizzare il proprio potere. Come e più di prima egli non vede alternativa per il principe nuovo, nella sua azione volta a mettere le «barbe e corrispondenzie», all'ottenimento del consenso popolare. Se si potesse scucire la sezione dedicata alle raccomandazioni da mettere in atto rivolte ad Appio e ai decemviri dal resto del capitolo e leggerla avulsa dal suo contesto, a nessuno potrebbe venire in mente di dire che Machiavelli non sia rimasto fermo anche in Discorsi I XL alle convinzioni espresse nel IX del Principe.

Una volta riconosciuto però che la critica svolta alla condotta del popolo e dei grandi, che mai avrebbero dovuto abbandonarsi all' 'errore» di rimettere l'intero potere nelle mani di un uomo solo, non comporta la riformulazione da parte di Machiavelli della sua precedente posizione sul principato, ci si deve rendere conto che la questione decisiva che va posta non riguarda cosa sia cambiato tra la stesura di Principe IX e quella di Discorsi I XL; ché è chiaro ormai che questi due testi, per la parte che concerne la fondazione del potere monocratico, recitano lo stesso copione. Il problema che va posto è piuttosto quello di vedere come incida sul significato da attribuire all'invito rivolto al principe a mantenere l'amicizia del popolo la contemporanea denuncia degli «errori» degli «umori» per avere, seppure involontariamente, favorito l'avvento del principato a spese della repubblica. Non ci vuole molto ad accorgersi, infatti, che l'identico precetto di farsi il popolo amico, come unica garanzia di successo per un principe appena arrivato al potere, che s'incontra in entrambi i capitoli qui esaminati, suoni assai diversamente da come suona in Principe IX, quando lo si ascolti insieme alle parole di biasimo rivolte a popolo e nobili per non avere operato per la preservazione delle istituzioni repubblicane di Discorsi I XL. All'interno del IX capitolo del trattato, la linea di condotta filopopolare prospettata da Machiavelli al principe pareva non generare alcun dubbio interpretativo. L'unilaterale angolo visuale del capitolo IX, con l'esclusione del punto di vista degli «umori» dall'orizzonte dell'analisi, sembrava essere l'espressione teorica di un preciso e rigoroso progetto politico. In quella sede l'indicazione di cercare l'amicizia del popolo si lasciava, quasi naturalmente, percepire come il patrocinio da parte di Machiavelli di un'alleanza organica tra popolo e principe, uniti dal comune obiettivo di rivitalizzare la compagine politica attraverso una svolta monarchica che ponesse fine alle lotte intestine che non consentivano il mantenimento della repubblica. Letto nelle pagine del Principe, quel «voltare la reputazione a uno» non faceva pensare in alcun modo ad un madornale «errore», del popolo o della nobiltà, che avrebbe finito per compromettere le basi 
della repubblica, ma alla giusta e necessaria medicina da mandare giù per risalire la china politica.

Ma Discorsi I XL ora sbarra la strada a quest'interpretazione, alla cui luce essa appare come il frutto di una carenza d'informazione. Mostrando unicamente i problemi di fondazione del potere del principe asceso alla testa dello stato con il favore di uno degli «umori», Principe IX induceva il lettore a scambiare la parte per il tutto e a concludere sbrigativamente che Machiavelli ravvisasse nel principato il necessario sodalizio da stringere tra popolo e sovrano, in cui fossero ugualmente garantiti gli interessi dei contraenti. La doppia prospettiva di Discorsi I XL, nel delineare il quadro completo della dialettica politica e qualificando come un «errore » che le parti sociali favoriscano il costituirsi di un potere monocratico, vieta invece di pensare che, rivolgendosi al principe, Machiavelli stia parlando il linguaggio del bene della comunità. Si ripropone quindi così la domanda già sopra formulata: se Machiavelli non riesce a vedere che un male politico nel principato, osservato con gli occhi della compagine sociale, dal quale né l'uno né l'altro « umore » può attendersi qualcosa di buono, qual è allora il significato in cui va effettivamente presa la regola politica secondo cui il potere regio può sostenersi e durare solo con l'appoggio del popolo, che Discorsi I XL ripesca direttamente da Principe IX?

Detto anche in altri termini, si tratta di capire a quale condizione era possibile per Machiavelli mantenere all'interno della stessa pagina due indicazioni tra di loro in apparente contraddizione. Quale operazione stava tentando che gli permetteva nel medesimo momento di bacchettare gli «umori», per essersi lasciati attrarre dalla sirena monarchica, e di ribadire che non c'è salvezza politica per un principe che non leghi a sé proprio quel popolo che da lui dovrebbe invece guardarsi, senza rischiare di smentirsi clamorosamente? La soluzione a questo problema non può che venire, ovviamente, dall'esatta messa a fuoco dell'operazione che Discorsi I XLintende svolgere. Affermando in apertura di capitolo di volere esaminare sia $\mathrm{i}$ «molti errori fatti dal senato e dalla plebe in disfavore della libertà», sia i «molti errori fatti da Appio, capo del Decemvirato, in disfavore di quella tirannide ch'egli si aveva presupposto stabilire in Roma», è peraltro Machiavelli stesso ad esplicitare al lettore il taglio con cui va presa la sua analisi, un taglio puramente tecnico, per così dire, rivolto ad indicare gli strumenti più efficaci per conseguire un determinato obiettivo politico. Sebbene non riesca a mascherare la sua personale preferenza per la repubblica, nondimeno l'intento programmatico con cui scrive il capitolo è quello di fornire una disamina dei mezzi più razionali al successo nella fondazione del potere, a prescindere dalla forma di stato che si vuole realizzare e da chi sia alle prese con la questione. È questa disposizione, che oggi si definirebbe di neutra avalutatività, che consente a Machiavelli alternativamente di calarsi nei panni dei diversi protagonisti della lotta politica e, sulla base del loro specifico obiettivo, apprezzarne gli atti e le scelte in ragione esclusiva della loro idoneità al fine da ciascuno perseguito. Egli può così, senza temere di contraddirsi, ad un tempo criticare gli «umori» sociali e l'aspirante principe. Ad entrambi i soggetti della lotta politica può impartire una severa lezione per avere agito in difformità con quello che era, e non poteva che essere, l'obiettivo che ciascuno si proponeva di raggiungere. «Errore» imperdonabile fu quello commesso dal popolo e dai grandi che, 
per riformare la repubblica, affidarono l'intero potere ad una sola magistratura; ma «errore» non meno grave fu quello compiuto da Appio, perché senza l'amicizia del popolo non vi era modo di fondare quella tirannide cui ambiva.

Premessa dell'analisi di Machiavelli è l'assunzione come legittimi dei progetti politici sia delle classi sociali sia di colui che vuole farsi principe. Egli ritiene un dato di fatto, che non merita nemmeno di essere posto a tema, la divergenza degli obiettivi politici delle diverse parti. Scontato che gli «umori» non possano avere altra preoccupazione che quella di conservare le istituzioni repubblicane, come altrettanto scontato è che chi ha la ventura di trovarsi nella posizione di Appio pensi a fare una «potestà assoluta». In quest'ottica, non è la valutazione della bontà in sé del fine politico ad occupare il centro del ragionamento di Machiavelli in Discorsi I XL, non perché la questione non gli importasse - sarebbe cosa davvero temeraria sostenerlo -, ritenendo l'una piuttosto che l'altra forma politica equivalenti, quanto perché egli parte dalla convinzione che su questo piano principe e classi sociali sono destinati a non incontrarsi mai. Muovendo dalla persuasione che principe e « umori» marciassero in direzioni opposte, lo spazio d'analisi che si apriva dinanzi a Machiavelli si poteva configurare, quindi, unicamente nei termini di un esame delle condizioni che rendono possibile la fondazione del potere, secondo i diversi orientamenti dei soggetti del confronto politico. Ed esso doveva avere una duplice articolazione per tenere conto dell'esigenza della collettività, volta a fondare o rifondare la repubblica, e del singolo individuo, desideroso di realizzare il proprio dominio personale. Nessuna delle due articolazioni poteva essere lasciata cadere entro una riflessione che si proponeva di esaurire l'intero spettro dei problemi che si pongono nella fondazione del potere. Completata, allora, la ricognizione del punto di vista della comunità, ed evidenziati gli «errori» commessi nell'intento di consolidare quella repubblica che non poteva che costituire il suo fine politico, rimaneva a Machiavelli ancora da considerare un lato della questione: quello che riguardava l'istituzione della "potestà assoluta». Così, come aveva fatto poco prima col popolo e coi grandi, gli toccava poi segnare in rosso gli «errori» compiuti da Appio che solo con l'aiuto dell' «universale», da cui infine inopinatamente per lui si scostò, avrebbe potuto attuare il proprio disegno assolutistico.

Il carattere tecnico dell'analisi svolta da Machiavelli in Discorsi I XL si rende pienamente palese, quando si consideri che il progetto tirannico di Appio viene condannato esclusivamente in contesti in cui la trattazione riguarda il punto di vista delle classi sociali, il cui interesse era indirizzato alla preservazione della repubblica. La tirannide di Appio viene criticata non in quanto tale, ma solo in riferimento allo scopo di chi si proponeva di conservare la repubblica. È a partire dal fine repubblicano che la tirannide si configura, in quanto forma-mezzo del suo trascendimento, negativamente. Arduo trovare parole più aspre di quelle con cui Machiavelli avverte il popolo dei pericoli cui va incontro quando, per sete di vendetta nei riguardi della nobiltà, dà «reputazione a uno perché batta quelli che elli ha in odio». In casi del genere non c'è modo di evitare, se quell' «uno sia savio », di finire sotto l'oppressione del regime tirannico. Egli, infatti, prima «attenderà insieme col favore del popolo a spegnere la nobiltà» e dopo, allorché più nulla avrà da temere da questo fronte, riserverà lo 
stesso trattamento al popolo, «nel quale tempo, conosciutosi il popolo essere servo, non abbi dove rifuggire». Con la plasticità tipica del suo linguaggio, Machiavelli non avrebbe potuto meglio spaventare il popolo e spingerlo ad un più prudente $\mathrm{e}$ moderato comportamento per non compromettere le istituzioni del «vivere libero ». $\mathrm{Ma}$, andando daccapo e attaccando il capoverso successivo, in cui è la posizione di Appio ad essere considerata, il suo tono muta del tutto. La condanna della tirannide aveva un senso rispetto alle classi sociali, ma non nei riguardi di chi invece proprio tiranno voleva farsi. Esaurita allora la prima parte della sua analisi, Machiavelli diventa prodigo di consigli per il tiranno, che non è più visto quale l'affossatore della repubblica, com'era stato solo un attimo prima, ma come colui che è alle prese con la fondazione del suo potere. Confrontandosi col suo progetto e con i suoi bisogni, Machiavelli non dimostra più di disapprovare le mire di Appio. Il suo caso, anzi, diventa il banco di prova sul quale sperimentare cosa si debba e non si debba fare per radicare solidamente un principato. Rispetto a questo nuovo problema, non può applicarsi quanto aveva detto poche righe sopra, che va bene per chi coltiva tutt'altro disegno. E se per gli «umori» la regola da osservare consisteva nel fare di tutto per sfuggire alla trappola monarchica nell'intento di riformare la repubblica, per Appio essa deve invece essere la messa in campo di tutto quanto può servire a «fermare » la sua «potestà assoluta».

In vista della quale "potestà assoluta», Machiavelli, in quello che sopra si è definito un supplemento d'indagine al capitolo I XL, cioè Discorsi I XLI, non prova alcun imbarazzo a lodare l' «astuzia» di Appio «nello ingannare la plebe, simulando d'essere popolare ${ }^{40}$. Dicendo che la plebe, nel concedere il proprio consenso ad Appio, si lasciò ingannare, Machiavelli ancora una volta fa trasparire la sua convinzione che l'affermarsi di un potere monarchico non rappresenti un'evoluzione politica vantaggiosa per il popolo. Ma avendo nel frattempo mutato punto d'osservazione e spostato il tiro sulle esigenze di Appio, l'«astuzia » mediante cui carpì il consenso del popolo non viene giudicata negativamente. Anzi, essa riceve un convinto apprezzamento perché si rivelò funzionale alla prospettiva politica perseguita dal decemviro. Ed anche il rilievo che poco oltre gli viene mosso, secondo cui sbagliò a «mutare in un subito natura, e di amico mostrarsi inimico della plebe $»^{41}$, conferma la logica della fondazione del potere che ispira Machiavelli nel trattare la vicenda di Appio. Egli avrebbe potuto sì scostarsi dal popolo, ma avrebbe dovuto farlo con «i debiti mezzi», cioè facendosi nuovi «amici» «innanzi che la diversa natura ti tolga de' favori vecchi », perché «altrimenti, trovandoti scoperto e sanza amici, rovini ${ }^{42}$. È vero che qui la regola sempre perentoriamente ribadita, secondo cui il potere monarchico può fondarsi solo sul popolo, sembra ammettere una deroga. Ma, in realtà, la modalità d'azione suggerita va intesa entro il carattere tecnico dell'analisi del caso di Appio condotta da Machiavelli. Passato a considerare il problema di indicare la prassi più efficace per la fondazione della «potestà assoluta», Machiavelli rimuove completamente le critiche di distruttore della repubblica che prima aveva rivolto ad Appio. Né si preoccupa oltre delle conseguenze che possono derivare per le istituzioni del «vivere libero» dalla creazione di un potere quale quello attribuito ai decemviri. Ciò che ora conta per lui è solo la puntuale e rigorosa analisi dei mezzi necessari alla 
fondazione di un potere assoluto. E nell'ansia di non trascurare alcun aspetto del suo comportamento, non si accorge neppure di proporre ad Appio, e a chi vuole seguirne i passi, una linea di condotta che entra in collisione con il principio generale che il successo nella fondazione del potere monarchico è garantito dal popolo.

Le due opposte indicazioni che s'incontrano nel capitolo I XL dei Discorsi - l'una che impone alle classi sociali di resistere all'avvento di un potere monarchico e, l'altra, che detta all'aspirante principe di avere il favore del popolo - , possono, dunque, coabitare nella mente di Machiavelli perché si riferiscono a situazioni e soggetti distinti che perseguono diversi e non relati tra di loro obiettivi politici. A ciascuno soggetto, in relazione alla sua situazione di partenza e al suo fine, Machiavelli vuole fornire una risposta. È, pertanto, l'abito dell' analista politico quello che, nella disamina della vicenda che portò alla costituzione del decemvirato a Roma, guida Machiavelli, la cui attenzione è tutta assorbita ad esaurire a $360^{\circ}$ i problemi che la fondazione del potere solleva. La materia di Discorsi I XL gli consentiva una trattazione completa della questione ${ }^{43}$. Egli poteva al contempo indicare la via da seguire a tutti i protagonisti della lotta politica in relazione alla loro diversa meta, con gli « umori» che dovevano assolutamente evitare di fare l'«errore » di conferire tutto il potere ad uno solo, perché «allora è che subito la tirannide surge », e chi vuole fondare una "potestà assoluta » che non doveva commettere l'«errore» di non avere amico il popolo, perché solo col suo appoggio è «più potente chi sforza che chi è sforzato ${ }^{44}$.

La doppia prospettiva di Discorsi I XL si rivela davvero preziosa. Essa rende possibile penetrare appieno il significato di Principe IX. L'aggiunta dell'angolo visuale degli «umori», che il commento della vicenda del decemvirato consente, porta alla luce ciò che nel capitolo del trattato era non detto, ma, non per questo, non pensato ${ }^{45}$. Si può così, infine, dare risposta alle questioni sollevate all'inizio della seconda parte del presente lavoro. In primo luogo, si dovrà allora riconoscere che Machiavelli non è mai giunto a teorizzare il principato a base di consenso popolare come rimedio politico per una repubblica in crisi. La repubblica è rimasta sempre e in ogni caso la sua prima scelta, rispetto alla quale il principato occupa un gradino politico inferiore. Al di là di ogni ragionevole dubbio, Discorsi I XL dimostra che né per il popolo né per la nobiltà il passaggio al principato possa costituire un progresso. Un tale passaggio si presenterebbe per gli «umori» comunque come un arretramento politico, non potendo il principato in alcun modo compensare la perdita della libertà, alimento di ogni avanzamento civile e garanzia contro l'oppressione. Che Machiavelli poi non abbia perso occasione per auspicare che un principe eserciti il potere non nella forma della violenza e dell'arbitrio particolaristico, ma garantendo l'ordine e la sicurezza sociale, che le sue pagine, in ogni sua stagione, siano colme di lodi per i sovrani che hanno governato per il bene della comunità, che la sua ammirazione per le «infinite constituzioni buone» del regno di Francia non sia mai scemata, non toglie che sia rimasto fermo alla convinzione che un popolo non possa trovare il proprio tornaconto nell'abbondare le istituzioni repubblicane a favore di quelle monarchiche. Tanto che ogni qualvolta dovesse favorire il trapasso dal primo regime politico al secondo, egli 
tornerebbe a stigmatizzarne le scelte come «errori». In base a Discorsi I XL non si può esitare a dire che per Machiavelli un popolo dovrebbe sempre preferire di vivere in un ordinamento repubblicano, per quanto le sue istituzioni possano essere sotto pressione. Quando dunque la prospettiva da cui si osserva la dialettica politica è data dall'interesse della comunità, il principato non regge il confronto con la repubblica, tra le due forme istituzionali non c'è partita.

In secondo luogo, Discorsi I XL, proprio affermando il primato della repubblica sul principato, ci spiega in che verso prendere il precetto che Machiavelli rivolge al principe di sempre cercare l'amicizia del popolo. Sebbene si pronunci per la repubblica contro il principato, Discorsi I XL non per questo rinuncia alla regola ampiamente tematizzata nel IX del Principe. Tra la composizione del Principe e dei Discorsi, Machiavelli non ha avuto alcun ripensamento, non ha rimesso in questione nei secondi una soluzione che gli pareva vincente nel primo: egli era ed è rimasto dell'opinione che è al popolo che deve guardare chi si accinga ad edificare un regime monarchico. Ma incontrare questa posizione accanto a quella che biasima gli «umori» per avere messo in pericolo le istituzioni repubblicane, affidando il potere ad uno solo, aiuta a determinarne e a circoscriverne esattamente il significato. La doppia prospettiva di Discorsi I XL si rivela decisiva per capire quale fosse l'intento di Machiavelli, quando stendeva le pagine del capitolo IX del Principe. Egli non si proponeva di tratteggiare i contorni del suo modello politico ideale, nel senso che ovviamente questo termine può avere per un pensatore realista ${ }^{46}$, e, balzando risolutamente oltre la tradizione del pensiero fiorentino contemporaneo, lasciarsi alle spalle un repubblicanesimo che aveva fatto ormai il suo tempo. Il suo scopo era più ristretto: fornire la risposta tecnica alla questione specifica delle risorse da mobilitare alla fondazione e preservazione di un principato ${ }^{47}$. E questo problema, come un'equazione matematica, aveva una sola risposta, qualsiasi fossero le convinzioni personali nei confronti del principato: il consenso del popolo. Ma Discorsi I XL, componendo tutte insieme le tessere del mosaico politico, circoscrive con precisione il senso di quest' indicazione che esprime esclusivamente l'interesse di chi vuole costituire una «potestà assoluta », e non certo quello del popolo ${ }^{48}$.

Che Machiavelli, d'altra parte, nella composizione del capitolo IX, e del Princi$p e$ in generale, guardasse al problema della fondazione del potere nella prospettiva dell'interesse del principe non stupisce, quando si riconoscano le sollecitazioni che il contesto storico-politico gli poneva e a cui si prefiggeva di dare risposta. In linea di massima, sciogliere i lacci che tanto strettamente legano la sua opera al suo tempo, per farne una riflessione di pura e astratta teoria, non pare essere la via più adatta per rendere un buon servizio al segretario fiorentino, immerso com'era fino al collo nelle faccende concretissime della politica italiana e fiorentina dei primi lustri del ' 500 . Quest'avvertenza vale in modo particolare per il Principe, e ancor di più per il suo capitolo IX che, nel concentrare, allusivamente, nel breve giro di poche pagine quasi un secolo della storia della città di Firenze, voleva trarre una lezione per il presente ${ }^{49}$. Per di più, questa lezione doveva essere congeniale al fine privato che Machiavelli si riproponeva di conseguire col suo opuscolo, la reintegrazione nell'ufficio di cancelliere col conseguente ritorno alla vita politica attiva che, per lui, aveva poco meno 
valore dell'aria che serve per respirare. Con la caduta della repubblica soderiniana e il ritorno dei Medici a Firenze l'agenda politica si riempiva di un solo punto, almeno per chi non intendesse battere in fuga dinanzi alla realtà. Fedele invece alla «verità effettuale della cosa», Machiavelli non poteva che misurarsi con gli equilibri e i rapporti di forza quali si erano determinati tra gli ultimi mesi del $1512 \mathrm{e} \mathrm{i} \mathrm{primi} \mathrm{del} 1513$. Pressato dall'attualità pubblica e personale, il suo impegno doveva necessariamente volgersi a stilare il promemoria che garantisse il successo del novello principe mediceo, mostrando al contempo quanto per costui potesse essere prezioso avere al proprio fianco un siffatto consigliere. Il momento storico imponeva un'assolutizzazione della prospettiva del principe, con conseguente spostamento fuori dell'orizzonte degli eventi di quanto della vicenda politica non si lasciava ricondurre a questa dimensione ${ }^{50}$. Con la repubblica ormai alle spalle, consegnata, quantomeno nel breve periodo, alla storia, attardarsi sugli «errori » di grandi e popolo e sulla forma politica che meglio poteva tutelarne gl'interessi, sarebbe suonato astrattamente accademico. L'urgenza della storia dettava a Machiavelli un altro programma: indicare i mezzi più adatti allo scopo di fondare un principato, con il suggerimento di cercare l'amicizia del popolo come via maestra per archiviare questo risultato.

Ma il solitario campeggiare di questo tema poteva provocare una percezione distorta dell'effettivo significato del Principe, inducendo a ritenere che Machiavelli avesse posto nel discorso sul principato molto più dei concreti e delle contingenti indicazioni per chi di fatto deteneva il potere a Firenze nel momento in cui scriveva. E il suo capitolo IX diventava così il perno su cui impiantare la tesi che egli fosse giunto alla conclusione che dalla crisi di una repubblica si uscisse solo col principato fondato sull'alleanza strategica tra principe e popolo. La duplice prospettiva analitica dei conflitti sociali di Discorsi I XL consente di mettere a fuoco con precisione quale sia l'effettiva posizione del principato e della repubblica nella costellazione di pensiero di Machiavelli e quale sia il senso esatto dell'operazione tentata da Principe IX. Quando Machiavelli solleva lo sguardo oltre i condizionamenti del momento storico e politico, per conferire alla sua riflessione uno spessore più squisitamente teorico, la repubblica gli si presenta come l'unica forma politica che può tutelare gli interessi e le aspirazioni degli «umori» sociali. Che si sia proposto di istruire il principe su come tenere lo stato, attraverso l'amicizia il popolo, non inficia che potesse comunque deplorare l'avvento del potere monocratico, considerandolo sempre un «errore » per chi vi approda da lidi repubblicani. 
Note

1. Senza alcuna pretesa di completezza, si indicano di seguito solamente le opere e gli autori che meglio, a giudizio di chi scrive, rappresentano la discussione che si è sviluppata intorno al capitolo IX del Principe, G. Sasso (Intorno a due capitoli dei Discorsi, in «La Cultura», IV 1966, poi in Studi su Machiavelli, Morano, Napoli 1967, pp. 111-159, Sogliono questi principati periclitare, in «La Cultura», XII 1974, pp.123-142, Principato civile e tirannide, in «La Cultura», XX 1982, pp. 213-275, e XXI 1983, pp.83-137, poi in Machiavelli e gli antichi, II, Ricciardi, Milano-Napoli 1988, pp. 351-490, Paralipomeni al principato civile, in Machiavelli e gli antichi, cit., II, pp. 511-540, Niccolò Machiavelli. Il pensiero politico, il Mulino, Bologna 1993), G. Cadoni (Intorno a due capitoli del Principe, in «La Cultura», IX, 1971, pp. 342-375, Machiavelli, Regno di Francia e "principato civile», Bulzoni, Roma 1974, Il principe e il popolo, in «La Cultura», XXIII, 1985, poi in Crisi della mediazione politica nel pensiero di N. Machiavelli, $F$. Guicciardini, D. Giannotti, Jouvence, Roma 1994, pp.93-165), V. Masiello (Critica sociale e riforma politica: la strategia del «principato civile», in Classi e stato in Machiavelli, Adriatica editrice, Bari 1971, pp. 49-124), M. Martelli, La logica provvidenzialistica e il capitolo XXVI del «Principe», in «Interpres», IV, 1981.82, pp. 262-384), F. Bausi (I 'Discorsi' di Niccolò Machiavelli. Genesi e strutture, Sansoni, Firenze 1985, pp.100-105, Machiavelli, Salerno, Roma 2005, pp. 200-16), G. Inglese (Il Principe di Niccolò Machiavelli, in Letteratura italiana, Einaudi, Torino 1992, Le Opere, vol. 1, a cura di A. Asor Rosa, pp. 909-13, poi in Per Machiavelli. L'arte dello stato, la cognizione delle storie, Carocci, Roma 2006, pp. 65-69), H. Baron (The republican citizen and the author of the Prince, in «English Historical review », 1961, pp. 217-253, poi in Machiavelli autore del Principe e dei Discorsi, Anabasi, Milano 1994, p. 11-65), P. Larivaille (Les 'Discours' et l'evolution de la pensée politique de Machiavel, Université de Paris X-Nanterre, 1977, pp. 79-86, poi La pensée politique de Machiavel. Les 'Discours sur la première décade de Tite-Live', Presses Universitaire de Nancy, Nancy 1982, pp. 77 ss., Nifo, Machiavelli, Principato civile, in «Interpres », IX (1989), pp. 150-195, «Amo la patria mia più dell'anima». La passione per Firenze nella genesi del 'Principe' e dei 'Discorsi', in AA.VV., Niccolò Machiavelli politico, storico, letterato, Salerno, Roma 1996, pp. 97-120, Il capitolo IX del Principe e la crisi del «principato civile », in AA.VV., Cultura e scrittura di Machiavelli. Atti del convegno di Firenze-Pisa 1997, Salerno, Roma 1998, pp. 221-239, Il capitolo IX del 'Principe' e la crisi fiorentina, in AA.VV., Studi di filologia e di letteratura italiana in onore di Gianvito Resta, Salerno, Roma 2000,pp. 345-54), M. Marietti (Le «principat civile» et son évolution dans l'ouvre de Machiavel in AA.VV., Le pouvoir monarchique et ses supports idéologiques aux XIVeXVIIe siècles, poi in Machiavelli, l'eccezione fiorentina, Cadmo, Fiesole 2005, pp. 76-85), M. Viroli, (From Politics to Reason of Srare. The Acquisition and Transformation of the Language of Politics 1250-1600, Cambridge University Press, Cambridge 1992, trad. it. Dalla politica alla ragion di stato . La scienza del governo tra XIII e XVII secolo, Donzelli, Roma 1994, pp. 89-95).

2. Il lettore che volesse farsi un'idea del travagliato e accidentato cammino interpretativo percorso da alcuni studiosi, che a più riprese sono intervenuti sull'argomento, non dovrebbe fare altro che allineare uno dopo l'altro cronologicamente gli scritti, riportati alla precedente nota, dedicati al principato civile.

3. In verità, l'affinità di Discorsi I XL con Principe IX è stata già segnalata: cfr. G. Cadoni, Machiavelli, Regno di Francia e "principato civile», cit., pp. 129-135, G. Inglese (a cura di), Niccolò Machiavelli, Discorsi sopra la prima deca di Tito Livio, Rizzoli, Milano 1984, 
p. 260 n., M. Marietti, Machiavelli, l'eccezione fiorentina, cit., pp. 82 ss., R. Rinaldi (a cura di), N. Machiavelli, I Discorsi sopra la prima deca di Tito Livio, Utet, Torino 1999, p. 633 n., F. Bausi (a cura di) N. Machiavelli, Discorsi sopra la prima deca di Tito Livio, Salerno, Roma 2001, p. 206 n., M. Martelli (a cura di), N. Machiavelli, Il Principe, Salerno, Roma 2006, pp. 167-68. Si spera che nel corso del presente lavoro si riesca a fare emergere con sufficiente chiarezza le ragioni per cui ci si discosta dalle posizioni sostenute dagli autori appena richiamati.

4. Le citazioni del Principe e dei Discorsi sono tratte dall'Edizione Nazionale delle opere di Niccolò Machiavelli: N. Machiavelli, Il Principe, a cura di M. Martelli, Salerno-Roma 2006, (d'ora in poi Il Principe, seguito dall'indicazione del capitolo e della pagina) e Niccolò Machiavelli, Discorsi sopra la prima deca di Tito Livio, a cura di F. Bausi, Salerno-Roma 2001, (d'ora in poi Discorsi, seguito dall'indicazione del libro, capitolo, e della pagina). Per evitare il moltiplicarsi delle note, si forniscono qui le indicazioni relative alle pagine di Principe IX, pp. 163-170, e di Discorsi I XL, vol. I, pp. 198-210, che nel corso del testo verranno citate.

5. Discorsi, I XVII, p. 110.

6. Cfr., per l'esame approfondito del significato di quest'espressione, P. Larivaille, Machiavelli, Nifo, «principato civile», cit., pp.175 ss.

7. Discorsi, I XLI, p. 211.

8. Discorsi I XXXV, pp. 172-73.

9. Discorsi, I XXXIII, p. 162.

10. Ibidem, p. 163.

11. Ivi.

12. Discorsi, I XXXIV, p. 168.

13. Ivi.

14. Ibidem, p. 169. Sulla differenza fra dittatura e decemvirato nella storia costituzionale e politica di Roma, cfr. C. Schmitt, Die Diktatur, Dunker \& Humbolt, Berlino 1964, trad. it., La dittatura, Laterza, Roma-Bari 1975, pp. 18-19.

15. È quanto pensano F. Bausi, I 'Discorsi' di Niccolò Machiavelli. Genesi e strutture, cit., pp. 100 ss., e M. Marietti, Machiavelli, l'eccezione fiorentina, cit., pp. 82 ss.

16. Cfr. G. Sasso, Principato civile e tirannide, in «La Cultura», cit., pp. 83 ss.

17. Sulle peculiarità della ricezione machiavelliana della teoria dell'anacyclosis, cfr. G. Sasso, La teoria dell'anacyclosis in Studi su Machiavelli, cit., pp. 161-222.

18. Discorsi, I II, p. 22.

19. Discorsi, I XXV, p. 135.

20. Ibidem, p. 137.

21. Su questa distinzione ha richiamato l'attenzione F. Bausi, I 'Discorsi' di Niccolò Machiavelli. Genesi e strutture, cit., pp. 81-2, rispetto al quale si offre qui una diversa interpretazione.

22. Discorsi, I XXVI, p. 138.

23. Ivi.

24. Ivi.

25. Ibidem, XXVI, p. 139.

26. Cfr. F. Bausi (a cura di), Niccolò Machiavelli, Discorsi sopra la prima la prima deca di Tito Livio, cit., p. 101, n.

27. Cfr. R. Rinaldi (a cura di), Niccolò Machiavelli, Discorsi sopra la prima deca di Tito Livio, cit., p. $565 \mathrm{n}$.

28. Ibidem, I IX, p. 65 .

29. Ivi.

30. Ibidem, I XVI, p. 106. 
31. La distinzione tra forme politiche del «vivere civile» e della «potestà assoluta» in relazione alla modalità con cui il potere è organizzato esclude che si possa definire «civile », come invece crede Sasso, Principato civile e tirannide, cit., pp. 217 ss., Machiavelli. Il pensiero politico, cit., pp. 385 ss., un principato in cui il potere è esercitato nell'interesse del popolo. Più brevemente e chiaramente, il principato filopopolare non è di per sé, cioè in quanto popolare, un principato «civile». Cfr. M. Marietti, Machiavelli, l'eccezione fiorentina, cit., p. 80.

32. Ibidem, II II, pp. 312-13.

33. Ibidem, I XVI, p. 103.

34. Ibidem, III III, p. 540. È da notare, peraltro, che anche all'interno dello stesso capitolo IXVI, tra quelli dei Discorsi che più si sogliono accostare a Principe IX, Machiavelli equipara, utilizzandoli indifferentemente, i concetti di tiranno, principe e re. Dopo avere scritto che chi fa una repubblica si fa «partigiani inimici [...] tutti coloro che dello stato tirannico si prevalevano, pascendosi delle ricchezze del principe», Machiavelli adduce come esempio di questa regola $\mathrm{i}$ «figliuoli di Bruto» che «con altri giovani romani» furono indotti a cospirare contro la repubblica «se non perché non si potevono valere strasordinariamente sotto i consoli come sotto i $r e »$. E ancora: richiamando la figura di Clearco che si sbarazzò dei nobili che lo avevano condotto al potere per accostarsi al popolo, Machiavelli la include nella categoria dei «principi che sono diventati della loro patria tiranni » (corsivo nostro).

35. Non si può condividere la recente interpretazione dell'ultimo capoverso di Principe IX proposta da G. Inglese, Per Machiavelli. L'arte dello stato, la cognizione delle storie, cit., p. 69. Sostiene Inglese che Machiavelli non inciterebbe il principe ad abbandonare l'«ordine civile» per l' «ordine assoluto», eliminando le magistrature del precedente regime repubblicano, "perché a Machiavelli non interessa tanto raccomandare al principe di passare da una forma di governo all'altra, quanto ricordare, una volta di più, "che a uno principe è necessario avere il populo amico, altrimenti non ha nelle avversità remedio" ». Ma non si capisce come Inglese ritenga che i principi, senza liquidare le magistrature, possano assicurarsi la fedeltà del popolo, dal momento che Machiavelli aveva attribuito proprio alla presenza dei «magistrati» la causa per cui «è più debole e più periculoso lo stare loro », perché nei momenti difficili «li cittadini e sudditi che sogliono avere e' comandamenti da' magistrati non sono in quelli frangenti per obedire a' sua ». Per la tesi secondo cui, invece, Machiavelli auspicherebbe il passaggio alla «potestà assoluta », cfr. H. Baron, Machiavelli autore del Principe e dei Discorsi, cit., p. 24, P. Larivaille, Les 'Discours' et l'evolution de la pensée politique de Machiavel, cit., p. 85, M. Marietti, Machiavelli, l'eccezione fiorentina, cit., p. 81 .

36. Pare, invece, che proprio all'apparenza esteriore si siano fermati i commentatori più recenti, la qual cosa ha determinato una serie d'interpretazioni insoddisfacenti del capitolo I XL dei Discorsi. Nel suo commento (N. Machiavelli, Discorsi, a cura di G. Inglese, cit., p. 260 n. 28-29), Inglese, dopo avere rilevato che la situazione descritta in I XL è «apparentemente identica a quella da cui, in Princ. 9, scaturisce il principato "civile" », spiega la definizione di tiranno data di Appio con il diverso grado di corruzione sociale dei due casi. Machiavelli riterrebbe tirannide quella che, sorgendo in un momento di assenza di corruzione, com'era la Roma del V secolo a.C., s'imporrebbe ad istituzioni repubblicane ancora vitali, e, invece, principato civile, con benefici effetti di risanamento sociale, quello che s'affermerebbe nella dilagante corruzione di Principe IX. Il punto è, però, che né nell'uno né nell'altro testo Machiavelli fa riferimento al ruolo che giocherebbe la corruzione nella dinamica socio-politica. Ed anche se si ammettesse che Appio è considerato tiranno per avere prematuramente soffocato una repubblica che aveva ancora un futuro davanti a sé, rimarrebbe da spiegare, poi, perché tale qualifica Machiavelli riservi anche a Cesare 
che, nonostante il favore popolare che gli viene sempre riconosciuto, operò in « una città corrotta» (Discorsi I X, p. 75. Cfr. anche I XVII, p. 109). Peraltro, lo stesso Inglese sembra avvertire le difficoltà della sua interpretazione quando precisa che Machiavelli, invitando Appio a coltivare l'amicizia del popolo, pare delineare « una sorta di variante positiva» della tirannide, col che, evidentemente, scomparirebbe ogni residua distanza tra Principe IX e Discorsi I XL.

Per F. Bausi, I 'Discorsi' di Niccolò Machiavelli. Genesi e strutture, cit., pp. 100 ss. e Niccolò Machiavelli, Discorsi sopra la prima deca di Tito Livio, (a cura di), cit., p. 206 n., seguito da M. Marietti, Machiavelli, l'eccezione fiorentina, cit., pp. 82 ss., Discorsi I $\mathrm{XL}$, sarebbe espressione di una stagione del pensiero machiavelliano connotata da acceso repubblicanesimo, per l'influenza subita dalla frequentazione, iniziatasi intorno al 1517, degli ambienti degli Orti Oricellari, che lo avrebbe indotto all'abbandono delle posizioni del Principe e a considerare quale tirannide ogni forma di potere monocratico. Per Bausi e la Marietti, insomma, quello che prima s'era presentato agli occhi di Machiavelli come principato civile, gli sarebbe poi apparso, una volta convertitosi alla repubblica, come intollerabile tirannide. Nel sostenere questo, però, Bausi non s'avvede di entrare a più riprese in contraddizione con se stesso. Nella sua monografia sui Discorsi, pp. 26 ss., scrive che la parte del capitolo I XVI dedicata a Clearco è stata con ogni probabilità composta all'epoca del Principe, di cui condividerebbe, in particolare, lo spirito animatore proprio del capitolo IX. Ma in quella sede Clearco viene definito «tiranno di Eraclea», col che si evince, all'opposto di quanto vorrebbe Bausi, e con lui Marietti che ne riprende le argomentazioni, che nello stesso arco temporale, quello della composizione del trattato, Machiavelli usava indifferentemente i termini principe e tiranno per indicare la medesima realtà della «potestà assoluta». Non basta: ripetutamente nel suo commento ai Discorsi (cfr., ad es., p. 101, n. 12, e p. 137, n. 9). Bausi fa notare l'equivalenza con cui Machiavelli adopera i termini principe e tiranno all'interno dei Discorsi, in accordo con una tendenza tipica del repubblicanesimo fiorentino quattrocentesco. E poiché anche nel suo più recente lavoro, Machiavelli, cit., pp. 163 ss. egli si mantiene fermo alla convinzione di una composizione estremamente dilatata dei Discorsi, avviati addirittura prima del 1510, non si capisce bene in base a cosa poi consideri la tirannide di Appio del capitolo I XL quale il ripudio della dottrina del principato del capitolo IX del trattato, quando, per la logica dei suoi stessi argomenti, avrebbe dovuto piuttosto concludere che Machiavelli lungo tutta la sua carriera di scrittore politico, che comprende naturalmente anche il Principe, ha impiegato come sinonimi principe e tiranno. Insomma Bausi, sostenitore della discontinuità teorica tra Principe IX e Discorsi I XL, produce poi buone ragioni a conferma della continuità d'impostazione fra i due testi.

37. G. Sasso, Principato civile e tirannide, cit., pp. 217 ss., Machiavelli, Il pensiero politico, cit., pp. 386 ss., V. Masiello, Crisi sociale e riforma politica: la strategia del «principato civile», in Classi e Stato in Machiavelli, cit., pp. 101 ss., G. Cadoni, Il principe e il popolo, in «La Cultura», cit., pp. 132 ss., G. Inglese, Per Machiavelli. L'arte dello stato, la cognizione delle storie, cit., pp. 65 ss., F. Bausi, Machiavelli, cit., pp. 208 ss.

38. A conforto di quanto si viene sostenendo, P. Larivaille, Il capitolo IX del Principe e la crisi del «principato civile», cit., p. 231, ha sottolineato che, quanto è esplicito in Discorsi I XL, deve ritenersi già in effetti pienamente operante anche in Principe IX: «lo scopo perseguito sia dall'uno che dall'altro umore non è - e non può che essere - di instaurare una monarchia: il che equivarrebbe per loro ad abdicare assurdamente alle proprie prerogative di cittadini. A quello che fanno principe, sia i grandi che il popolo non mirano ad altro che ad affidare una preminenza, un'autorità dalla quale si aspettano che le istituzioni cittadine vigenti vengano non soppresse, ma solo interpretate, usate e per così dire piegate nel senso dei loro interessi ed appetiti ». 
39. È questa la convinzione che ispira anche Discorsi I XVII e XVIII. Nel primo, vengono esaltate quelle figure di «principi », quali il tebano Epaminonda, che non hanno approfittato della loro posizione per operare il passaggio al principato, ma che, anzi, hanno lavorato per il mantenimento delle istituzioni repubblicane. Vengono invece biasimati coloro che come Cesare hanno approfittato della lacerazione del tessuto sociale non per ricucirne gli strappi, ma per accentuarne il disfacimento e imporre il proprio potere personale. Il secondo è dominato dal timore che, nelle repubbliche «corrottissime», proprio il passaggio al principato sia inevitabile, con la relativa decadenza che ciò comporta. Dovendosi ricorrere a mezzi estremi per estirpare la corruzione, dove si troverà un uomo, si domanda Machiavelli, che userà «quella autorità bene, che egli ha male acquistata » per riordinare e non per spegnere la repubblica? In entrambi i capitoli, il principato non è auspicato per i suoi eventuali effetti risanatori, che Machiavelli non vede, presentandosi esso, piuttosto, come il sicuro segno del declino politico di un popolo. E la stessa introduzione di una «potestà quasi regia » nell'ordinamento costituzionale non può essere interpretata, come ha riproposto anche di recente Inglese, Per Machiavelli. L'arte dello stato, la cognizione delle storie, cit., p. 139, come se Machiavelli fosse giunto «a un passo dal riconoscere nel principato » il solo possibile rimedio alla corruzione di una repubblica. All'opposto, la «potestà quasi regia» esprime l'estrema resistenza alla transizione al principato che, anche quando fosse inevitabile, andrebbe visto come espressione di decadimento politico.

40. Discorsi I XLI, p. 211.

41. Ivi.

42. Ibidem, p. 212.

43. Il non avere colto il taglio tecnico alla base dell'impianto analitico di Machiavelli ha impedito a G. Cadoni, Regno di Francia e «principato civile », cit., p. 135, di comprendere come appunto possano coesistere nella stessa pagina « la convinzione che solo il favore del popolo assicuri al "tiranno" un saldo sostegno » e «la tesi secondo cui il potere sorto sulla rovina della libertà repubblicana dà necessariamente luogo all'indiscriminata oppressione di tutti i cittadini».

44. Per riprendere la distinzione operata da M. Viroli, si potrebbe dire che Discorsi I XL sia un eccellente esempio di quell' «arte dello stato», quale precettistica tecnica rivolta al «principe nuovo per conquistare e mantenere il suo stato », che si andrà imponendo nel corso del '500, costituendo il fondamento dell'idea di ragion di stato, sull' «arte della politica», come filosofia civile mirante al perseguimento del bene della comunità, che peraltro, secondo lo studioso, sarebbe comunque prevalente nella riflessione del segretario fiorentino. Con la precisazione che nel capitolo del commento liviano in oggetto, l' « arte dello stato » è utilizzata quale criterio analitico anche nei riguardi delle classi sociali e non del solo principe. La quale constatazione, tuttavia, indurrebbe a ritenere che il rapporto fra «arte dello stato » $\mathrm{e}$ «arte della politica» in Machiavelli s'intrecci più strettamente di quanto pensi Viroli. Cfr. Dalla politica alla ragion di stato. La scienza del governo tra XIII e XVII secolo, cit., pp. 83-108. Sulla presenza nella riflessione di Machiavelli di una concezione «tecnica» della politica, si possono ancora tenere presenti le pagine di L. Russo, Machiavelli, Laterza, Bari 1975, pp. 24 ss., nonché le brevi, ma penetranti osservazioni di C. Schmitt, La dittatura, cit., pp. 20 ss.

45. A ben considerare, non si vede cosa possa autorizzare ad affermare che Principe IX, anche prescindendo dal confronto con Discorsi I XL, ponga lo sbocco monocratico come la conseguenza necessaria, inevitabile e positiva della crisi delle istituzioni repubblicane, dal momento che Machiavelli scrive che dal contrasto degli «appetiti» di grandi e popolo «nasce nelle città uno de' tre effetti, o principato, o libertà, o licenza». «Principato», «libertà », cioè repubblica e «licenza », anarchia, vengono situati sullo stesso piano, quasi che Machiavelli volesse sottolineare l'identica probabilità che abbiano di materializzarsi, 
senza che, per di più, alcun dato testuale possa consentire di ritenere che sia espressa una preferenza per il principato. Che venga poi svolta solo l'ipotesi monarchica, si spiega coll'oggetto specifico del trattato, che programmaticamente rinuncia all'esame di quanto fuoriesca dall'ambito del principato. Anche ad attenersi, dunque, al solo capitolo nono, con tutto il suo non detto, avrebbe dovuto, e dovrebbe, ispirare maggiore prudenza interpretativa, evitando troppo perentorie conclusioni sulla conversione di Machiavelli al principato, popolare, quale auspicabile approdo per una repubblica allo stremo.

46. Per la centralità di Machiavelli nella fondazione del realismo politico moderno, cfr. P.P. Portinaro, Il realismo politico, Laterza, Roma-Bari 1999.

47. Si ritiene che si debba capovolgere la tesi di G. Sasso, Intorno a due capitoli dei Discorsi, in Studi su Machiavelli, cit.,p. 157, per la quale la proposta repubblicana che si affaccerebbe nel Discursus florentinarum rerum, nascerebbe da un restringimento della prospettiva analitica machiavelliana che, da italiana facendosi fiorentina, rinuncerebbe alla soluzione più ardita e moderna, rappresentata dal principato teorizzato nel Principe, a favore di quella repubblicana legata alla tradizione della democrazia comunale. La proposta repubblicana del Discursus florentinarum rerum va vista, al contrario, come la riproposizione di una convinzione di fondo di Machiavelli, temporaneamente messa tra parentesi solo per la particolare e contingente situazione politica determinatasi nel 1513 e negli anni immediatamente successivi. La tesi di Sasso può farsi risalire ad un giudizio che ha contribuito a far largamente circolare F. Chabod, Scritti su Machiavelli, Einaudi, Torino 1982, pp. 105 ss., secondo cui il segretario fiorentino aveva in mente per l'Italia il modello offerto dalle grandi monarchie nazionali che si stavano affermando in Europa, in primis quello costituito dal regno di Francia. Ma, come ha acutamente osservato F. Gilbert, nel suo intimo Machiavelli è sempre rimasto repubblicano e «non disperava del futuro degli stati cittadini [...] La passione con cui Machiavelli discute ogni particolare della politica romana era stimolata dalla sua convinzione che Roma era stata ciò che Firenze avrebbe potuto essere, e forse poteva ancora diventare », Machiavelli e Guicciardini. Politics and history in Sixteenth-Century Florence, Princeton University Press, 1965, trad. it., Machiavelli e Guicciardini, Einaudi, Torino 1970, p. 158. Sul repubblicanesimo di Machiavelli, cfr. anche R. von Albertini, Das florentinische Staatsbewusstsein im Übergang von der Republik zum Prinzipat, A. Francke AG Verlag, Berna 1955, trad. it., Firenze dalla repubblica al principato, Einaudi, Torino 1995, p. 52.

48. La stessa logica, quella di fornire le indicazioni tecniche per la fondazione del potere di un principe, è operante anche nel capitolo I XVI che, come si è avuto modo già di dire sopra, è ritenuta la pagina dei Discorsi più affine a Principe IX per la riproposizione del tema del principato filopopolare. Il tema dichiarato del capitolo è costituito dalle difficoltà che un popolo, «uso a vivere sotto uno principe », incontra a mantenere la libertà una volta raggiuntala. In realtà la trattazione svolge poi in modo indipendente e distinto due questioni: quella che riguarda chi «prende a governare una moltitudine [...] per via di libertà » e quella che concerne chi prende a governare «per via di principato ». Senza entrare nel merito del rapporto fra le due forme di governo ma, da consigliere imparziale, interessato a fornire indicazioni che possono solo favorire il successo dell'operazione di fondazione dello stato, Machiavelli impartisce quindi la sua lezione sul regime change. $\mathrm{E}$ se chi «prende a governare [...] per via di libertà deve sbarazzarsi dei figliuoli di Bruto », cioè dei partigiani del regime monarchico, chi costituisce un principato deve « guadagnarsi uno popolo che gli fosse inimico ». In questa prospettiva analitica, in cui si prescinde da ogni giudizio di valore sulle due forme di governo fondamentali, Machiavelli, attraverso Clearco, tiranno di Eraclea, prospetta la ricetta che aveva presentato ad Appio sulla necessità di farsi il popolo amico. È istruendo il principe e non il popolo che Machiavelli invita il primo a stringere un legame positivo col secondo. Ma nulla del ragionamento di Machiavelli 
lascia intendere che la condizione politica monarchica rappresenti per il popolo, rispetto alla repubblica, un passo avanti o una transizione augurabile. Anzi, se un'indicazione si deve trarre dal capitolo I XVI, essa è di carattere opposto a questa. Delle «due cose» che un popolo desidera e che un principe deve soddisfare per averne in cambio il consenso, la vendetta verso i suoi oppressori e la libertà, la seconda non può essere concessa, per l'evidente dissoluzione della monarchia che implicherebbe. Il principe può solamente surrogare la libertà con la sicurezza. Ma se garantire la sicurezza giova al principe nell'azione fondativa del suo potere, essa è pur sempre un passo indietro per il popolo che perde la libertà. D'altra parte, che la logica sottesa alle considerazioni svolte da Machiavelli sulla nascente monarchia abbia un carattere puramente tecnico e non sia ispirata al proposito di favorire un'alleanza strategica tra principe e popolo, è confermato dal seguente passaggio, sul quale solitamente si preferisce glissare: «Perché in tutte le republiche, in qualunque modo ordinate, ai gradi del comandare non aggiungano mai quaranta o cinquanta cittadini: e perché questo è piccolo numero, e è facil cosa assicurarsene, o con levargli via, o con fare loro parte di tanti onori che, secondo le condizioni loro, e' si abbino in buona parte a contentare». Indicare al principe il modo di «assicurarsi » di quei «quaranta o cinquanta cittadini » che potrebbero insidiare il suo potere, non pare certo espressione di una volontà che, nell'indicare le condizioni del radicamento del reggimento monarchico, abbia di mira la tutela dell'interesse del popolo. Si veda anche Discorsi, III III, pp. 605-06.

49. Sul carattere fiorentino del capitolo IX ha particolarmente insistito P. Larivaille, Les 'Discours' et l'evolution de la pensée politique de Machiavel, cit., pp. 79 ss. Il motivo fiorentino del principato civile, peraltro, ricorre in tutti lavori che lo studioso ha dedicato al tema, e per i quali si rinvia sopra alla nota 1.

50. Ci pare che veda bene M. Viroli, Dalla politica alla ragion di stato. La scienza del governo tra XIII e XVII secolo, cit., p. 89, quando scrive che Machiavelli aveva nel capitolo IX soprattutto l'obiettivo polemico di criticare quella vasta pubblicistica fiorentina che indicava nel sistema messo in piedi da Cosimo a partire del 1434, con la sua regola fondamentale di «governare con "civiltà", ovvero controllare le istituzioni cittadine lavorando dietro le quinte», il modello cui si sarebbero dovuti attenere anche dopo il 1513 i Medici, e di proporre in alternativa, per fermare il loro stato, la più solida via di «salire dall'ordine civile allo assoluto». 